\title{
ESTRUCTURA EMPRESARIAL COMPETITIVIDAD EN MÉXICO
}

\section{Bussines structure and competitiveness in Mexico}

\author{
José Guadalupe Vargas-Hernández ${ }^{1}$ \\ Martha Lizbeth Bautista Ramírez²
}

1. Profesor Investigador miembro del Sistema Nacional de Investigadores

Departamento de Administración Centro Universitario de Ciencias Económico-Administrativas Universidad de Guadalajara (México) E-mail: jvargas2006@gmail.com

2. Maestría en Negocios y Estudios Económicos Centro Universitario de Ciencias Económico Administrativas. Universidad de Guadalajara (México) E-mail: martha.liz.bautist@gmail.com

\section{Citación sugerida:}

Vargas-Hernández, J.G. y Bautista Ramírez, M. L. (2016). Estructura empresarial y competitividad en México. $3 C$ Empresa: investigación y pensamiento crítico, 5(3), 26-53. DOI: <http://dx.doi.org/10.17993/3cemp.2016.050327.26-53/>. 


\section{RESUMEN}

Este trabajo tiene como objetivo analizar la estructura, características y desempeño empresarial en las 32 entidades federativas de la República Mexicana, para determinar sus niveles de competitividad. Se parte de un estudio previo realizado por Unger, Flores e Ibarra (2013), dentro del cual se aplica un modelo para medir la competitividad empresarial. Para hacerlo, se toman como variables el salario y el valor agregado en el modelo. Los resultados de la investigación confirman la hipótesis de que la competitividad de los Estados puede determinarse través de la estructura empresarial, la productividad y por lo tanto una ventaja salarial más elevada.

\section{ABSTRACT}

This paper aims to analyze the structure, characteristics and business performance in the 32 states of Mexico, to determine their levels of competitiveness. It follows a previous study by Unger, Flores and Ibarra (2013), in which a model is applied to measure business competitiveness. The variables that are taken are the salary and the value added in the model to measure competitiveness. The research results confirm the hypothesis that the competitiveness of states can be determined by the business structure, productivity and therefore higher wage advantage.

\section{PALABRAS CLAVE}

Estructura empresarial; México; salario; valor agregado

\section{KEY WORDS}

Business structure; Mexico; salary; value added 


\section{INTRODUCCIÓN}

El objetivo de este trabajo de investigación es determinar la estructura, características y desempeño empresarial de la Pequeña y Mediana Empresa (MiPyME) y la Gran Empresa (GE) al interior de la república Mexicana de las 32 entidades federativas, con el fin de observar que Estados son o no competitivos. Se parte de un estudio previo realizado por Unger, Flores e Ibarra (2013), dentro del cual se aplica un modelo para medir la competitividad de los estados, y se crean dos sub-grupos, el primero engloba a los estados competitivos y el segundo a los estados no competitivos. La aplicación de este modelo se hará para todos los estados del país clasificando la MiPyME y la GE para conocer el comportamiento empresarial y demostrar si estos Estados son o no competitivos.

Para fines de este trabajo se hace un análisis de cada una de las actividades económicas de los Estados de México, seleccionando el total por ramas industriales. Adicionalmente, se seleccionaran las variables de interés en el estudio (unidades económicas, personal ocupado total, personal remunerado, total de remuneraciones, producción bruta total, valor agregado censal bruto, formación bruta de capital fijo, inversión total, acervo total de activos fijos, depreciación total de activos fijos). Se plantea como objetivo específico:

A) Describir la competitividad en función de los salarios y el valor agregado.

Por ello, en la presente investigación, se plantea describir la relación que existe entre el desempeño de las empresas y la competitividad de los estados, estableciendo las siguientes hipótesis:

1) $\mathrm{H}^{0} \mathrm{El}$ desempeño de las empresas determinan el nivel de competitividad de los estados.

2) $\mathrm{H}^{1}$ La competitividad de los estados del país se mide a través de la estructura empresarial MiPyME. Metodología

El aporte metodológico de esta investigación para el cumplimiento de los objetivos del estudio, estuvo centrado en el diseño de instrumentos abordados en el trabajo de campo. Se acudió al uso de técnicas de investigación como la observación, el cuestionario, la entrevista, las fichas, y el análisis de contenido

El cuestionario empleado como instrumento buscó proponer un sistema integral de información financiera, que les permita a Las Asociaciones Cooperativas Bancos Comunales del Municipio Díaz, Estado Nueva Esparta mejorar de manera significativa sus gestiones públicas. Dicha propuesta estuvo sustentada en un Modelo Operativo para satisfacer las necesidades de organización de las mismas. De esta forma los resultados de la investigación se apoyaron en la aplicación de técnicas de investigación válidas en el medio como fue el cuestionario cerrado y una encuesta de opinión. 


\section{LA ESTRUCTURA EMPRESARIAL}

En México, el panorama general de los estudios sobre la estructura empresarial, empresarios, comerciante etc. presenta características muy heterogéneas (Romero, 2003: 805). El presente trabajo se enfoca en la clasificación de las empresas por su tamaño, ya que se describe el comportamiento de la MiPyME y la GE para identificar qué Estados de la República Mexicana son competitivos. Así mismo, se describe la competitividad en función de los salarios y el valor agregado que se genera en cada entidad federativa.

A partir de los resultados de los Censos Económicos 2009, el Instituto Nacional de Estadística y Geografía (INEGI), permite la estratificación de los establecimientos por personal ocupado. Las empresas se pueden clasificar dependiendo de su sector, ya que estas pueden ser comerciales, industriales o de servicio. Así mismo, las empresas se clasifican como micro, pequeñas, medianas o grandes por el número de empleados que laboran dentro, el volumen de su producción y por sus ventas medidas en salarios mínimos anuales.

En muchas regiones, la PyME es la única fuente de empleo y de renovación económica (De la O Hernandez, Ramírez Ruiz, Ayala Rodríguez y Bonilla (2007). Las PyMEs atraviesan cambios trascendentales debido a la globalización y la apertura económica que ha experimentado nuestro país, ya que son un elemento fundamental para el desarrollo económico. Constituyen una parte importante de la economía nacional por los acuerdos comerciales que México ha tenido en los últimos años, contribuyendo en un alto porcentaje la productividad, competitividad y sustentabilidad, así como el incremento de participación en los mercados, en un marco de crecientes encadenamientos productivos que generan mayor valor agregado nacional.

\section{COMPETITIVIDAD}

El concepto de competitividad es muy amplio, ya que existe un uso muy generalizado que se hace del mismo y de la abundante literatura sobre el tema, y existe ambigüedad en el significado que se le da. La competitividad se asocia con la rentabilidad, productividad, costos, valor agregado, participación del mercado, exportación, innovaciones tecnológicas, entre otros (McFetridge, 1995). Unger et al. (2013) miden la competitividad de los estados a través de dos indicadores económicos fundamentales, la productividad laboral y los salarios. Cada uno de estos indicadores están asociados a las actividades económicas de cada estado y de esta manera clasifica y agrupa los estados según sus condiciones de competitividad para orientar medidas de política.

El presente trabajo se basa en el estudio de la productividad laboral y los salarios promedio como variables explicativas de la competitividad revelada de los estados, haciendo en paralelo una observación sobre las especializaciones que las entidades poseen en sus respectivas industrias. Aunado a esto, se calculará el índice de competitividad relevada enfocada al tamaño de empresa y por actividad económica. Así mismo, se calculará el nivel 
de concentración industrial e índices de Herfindahl-Hirschman (IHH) e Índice de Dominancia (ID).

\section{METODOLOGÍA}

El análisis de la competitividad se hace en consideración de dos indicadores económicos fundamentales, la productividad laboral y los salarios, con el propósito de distinguir a los estados en cuanto a dos criterios:

A) Por tipos de Estado según sus condiciones de competitividad - competitivos y no competitivos.

B) Por sub-grupos en que cada Estado refleja sus condiciones de productividad y salarios.

La variable a utilizar es el valor Agregado Censal Bruto (Miles de Pesos).

El Plan de procesamiento de la información se elaborará índices para medir la competitividad, en función de salarios y valor agregado, y competitividad por estado y estrato (MiPyME - GE).

\subsection{ESTIMACIÓN DE VENTAJA POR PRODUCTIVIDAD LABORAL Y VENTAJA POR SALARIO}

El estudio de la competitividad regional empieza con la productividad laboral, pero tiene que ir más allá de la descripción del resultado, pues es necesario estudiar otras dimensiones características de todo territorio (Martin y Sunley, 2003). En este trabajo se profundiza en la versión de competitividad de las actividades y entidades. La estimación consiste en integrar dos elementos fundamentales de la competitividad económica: la productividad laboral relativa a la del país (ventaja revelada por productividad), y el nivel salarial relativo que se asocia como indicador del empleo de mano de obra con mejor calificación (ventaja revelada salarial).

Las variables seleccionadas para la estimación de ventaja por productividad laboral y ventaja por salario respecto al censo económico 2009 son las siguientes:

a. Valor agregado censal bruto.

b. Personal remunerado.

c. Total de remuneraciones.

Para la estimación de productividad laboral de cada actividad económica por estado se dividió "VA" (Valor Agregado Censal Bruto) entre "L" (Personal Remunerado). Para la estimación de ventaja por salario de cada estado y cada actividad económica se dividió "W" (Total de remuneraciones) entre "L" (Personal Remunerado). 


\section{2. ÍNDICE DE COMPETITIVIDAD RELATIVA O REVELADA (ICR)}

Con datos de las variables de los Censos Económicos del INEGI (2009) (valor agregado censal bruto, Personal Remunerado y total de remuneraciones) se calculan por separado cada una de las ventajas reveladas del estado. Primero se obtiene el producto por trabajador para el estado $\left(\frac{\mathrm{VA}}{\mathrm{L}}\right)$ Edo. y se divide por la misma razón pero a nivel nacional $\left(\frac{\text { VA }}{\mathrm{L}}\right)$ País. Lo mismo sucede para las remuneraciones promedio $\left(\frac{\mathrm{W}}{\mathrm{L}}\right)$ Edo. entre $\left(\frac{\mathrm{W}}{\mathrm{L}}\right)$ País. Ambas medidas en conjunto nos acercan a la competitividad relativa o "revelada" $C^{* *}$ de la actividad en el estado.

En otras palabras la competitividad de las actividades en cada entidad puede verse en la comparación de cada actividad respecto de dos factores:

A) La estimación de la productividad laboral de cada actividad en el Estado con respecto a la misma a nivel nacional (VA/L).

B) La comparación del salario promedio de la actividad en el Estado con el promedio salarial nacional de dicha actividad (W/L).

La siguiente fórmula expresa la suma de ambas ventajas en relación a las actividades de cada estado.

Fórmula 1: Suma de ambas ventajas en relación a las actividades de cada estado.

$$
C_{\text {act }}^{* 8}=\left(\frac{V A}{L}\right)+\left[\left(\frac{W}{L}\right)-1\right]=\left[\left(\frac{\frac{V A}{L_{\text {edo }}}}{\frac{V A}{L_{\text {pais }}}}\right)\right]+\left[\left(\frac{\frac{W}{L_{\text {edo }}}}{\frac{W}{L_{\text {pais }}}}\right)-1\right]
$$

\section{A. Análisis de los resultados obtenidos}

A continuación, se observan los índices de ventaja por productividad, salario y competitividad en la tabla 1, relativos para cada uno de los estados de la Republica correspondientes al censo económico 2009, (INEGI, 2009).

Tabla 1: Índices de ventaja por productividad - salario y Competitividad relativa por entidad federativa de los Estados Competitivos.

\begin{tabular}{|c|c|c|c|}
\hline Entidad & $\begin{array}{c}\text { Ventaja por } \\
\text { productividad } \\
\text { (VA/L*) }\end{array}$ & $\begin{array}{c}\text { Ventaja por } \\
\text { salario (W/L*) }\end{array}$ & $\begin{array}{l}\text { Competitividad } \\
\text { relativa }\left(\mathrm{C}^{* *}\right)\end{array}$ \\
\hline Nuevo león & 1.10129116 & 1.12002702 & 1.221318184 \\
\hline Distrito federal & 1.364488434 & 0.44693109 & 0.811419521 \\
\hline Baja california & 1.012005606 & 0.36235269 & 0.374358298 \\
\hline Querétaro & 1.124042443 & 1.11073543 & 1.23477787 \\
\hline Sonora & 0.940678309 & 1.01921129 & 0.959889597 \\
\hline Baja california sur & 1.171728391 & 0.37230465 & 0.544033042 \\
\hline
\end{tabular}




\begin{tabular}{|l|l|l|l|}
\hline Coahuila & 0.740608869 & 0.3327698 & 0.073378671 \\
\hline Chihuahua & 0.874791711 & 0.3341344 & 0.208926115 \\
\hline México & 0.949551927 & 0.2932799 & 0.242831828 \\
\hline Jalisco & 0.914535955 & 0.32997744 & 0.244513398 \\
\hline Tamaulipas & 1.016609742 & 0.97542984 & 0.992039579 \\
\hline
\end{tabular}

Fuente: Elaboración propia con datos del Censo Económico 2009, INEGI.

El índice de competitividad relativa se obtiene a través de los datos de ventaja por productividad y ventaja por salario. Querétaro es el estado con mayor competitividad relevada, seguido de Nuevo León, Tamaulipas, Sonora, Distrito Federal, Baja California Sur, Baja California, México y Jalisco se mantienen a la par, seguidos de Chihuahua y, finalmente, Coahuila.

Tabla 2: Comparación de Valor Agregado entre Personal Remunerado (2009).

\begin{tabular}{|l|c|c|c|}
\hline \multicolumn{4}{|c}{ Promedio de VA/L } \\
\hline Entidad & MiPyME & Grande & Total General \\
\hline Nacional & 17999.62 & 950.45 & 13796.80 \\
\hline Nuevo león & 4966.72 & 430.51 & 4059.48 \\
\hline Distrito federal & 31918.07 & 738.54 & 24161.97 \\
\hline Baja california & 237.88 & 507.77 & 280.89 \\
\hline Querétaro & 348.77 & 311.77 & 342.81 \\
\hline Sonora & 281.27 & 733.98 & 360.43 \\
\hline Baja california sur & 122.73 & 380.01 & 147.16 \\
\hline Coahuila & 195.23 & 391.29 & 223.24 \\
\hline Chihuahua & 251.89 & 264.49 & 254.16 \\
\hline México & 268.07 & 456.79 & 303.64 \\
\hline Jalisco & 268.36 & 367.66 & 286.79 \\
\hline Tamaulipas & 298.72 & 601.78 & 344.18 \\
\hline
\end{tabular}

Fuente: Elaboración propia con datos del Censo Económico 2009, INEGI.

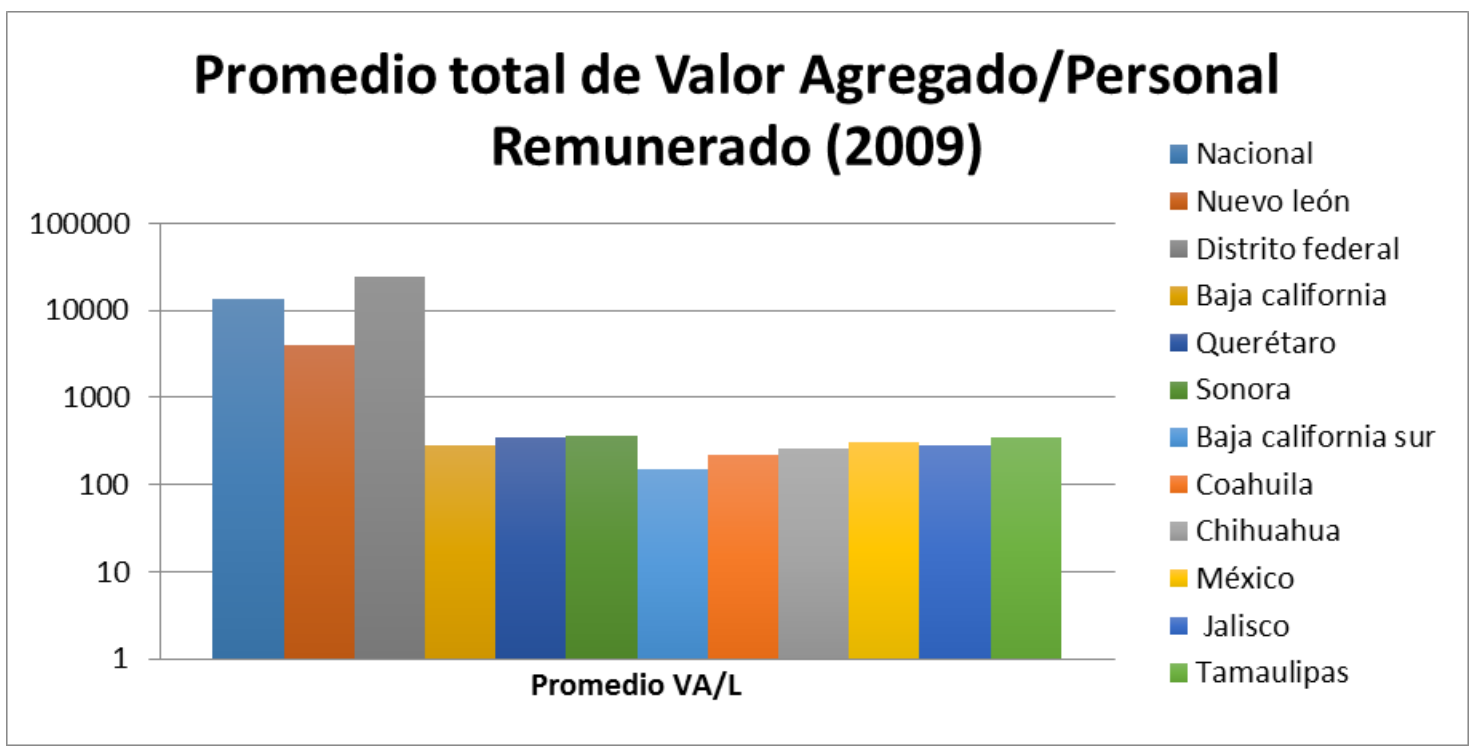

Figura 1: Promedio total de Valor Agregado/Personal Remunerado (2009). Fuente: Elaboración propia con datos del Censo Económico 2009, INEGI. 
La tabla 2 y la figura 1 exponen el comportamiento del Valor Agregado/ Personal Remunerado a nivel nacional y de Nuevo León, Distrito Federal, Baja California, Querétaro, Sonora, Baja California Sur, Coahuila, Chihuahua, Edo. De México, Jalisco y Tamaulipas. El Distrito Federal presenta un mayor nivel competitivo de Valor Agregado, le sigue el promedio Nacional, seguido de Nuevo León, Sonora, Tamaulipas, Querétaro, Edo. De Méx., Jalisco, Baja California, Chihuahua, Coahuila y finalmente, el Estado de Baja California Sur.

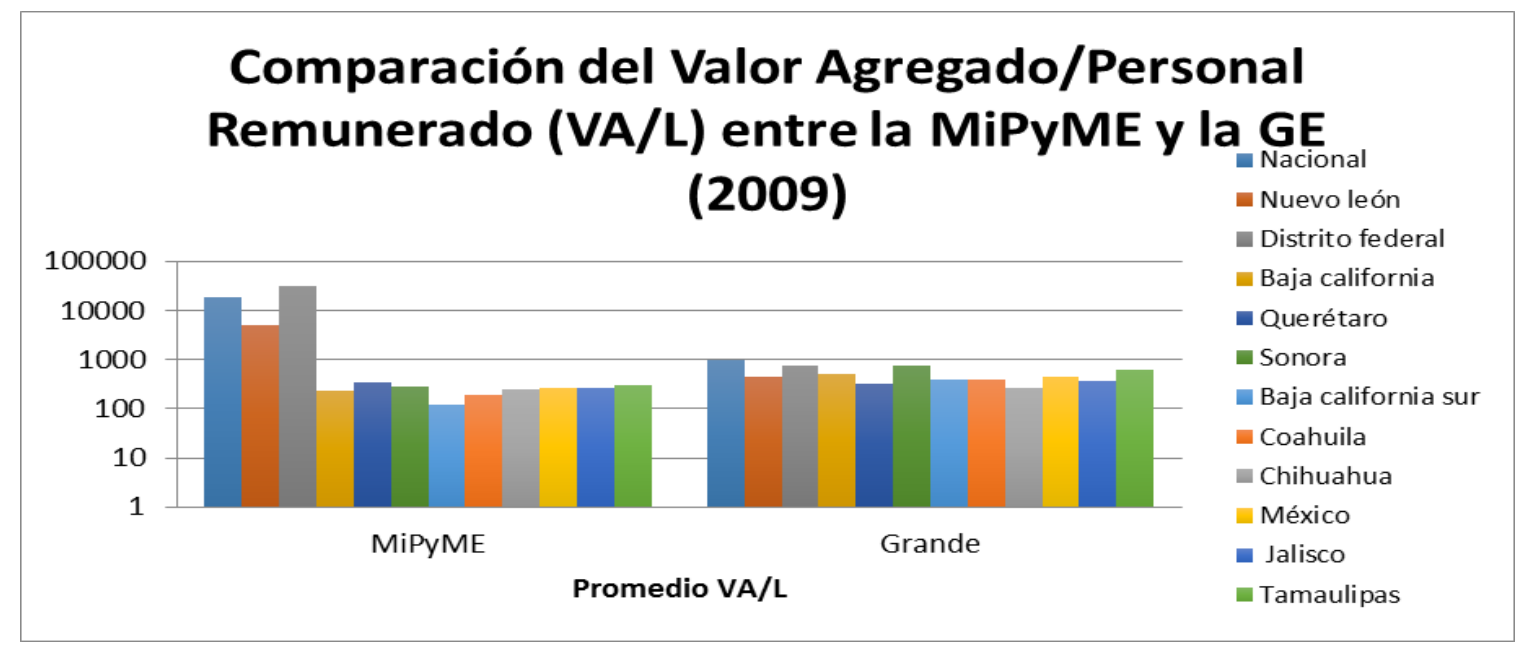

Figura 2: Comparación del Valor Agregado/Personal Remunerado (VA/L) entre la MiPyME y la GE (2009).

Fuente: Elaboración propia con datos del Censo Económico 2009, INEGI.

La figura 2 muestra una comparación del valor agregado/ personal remunerado entre la MiPyME y la Gran Empresa de Nuevo León, Distrito Federal, Baja California, Querétaro, Sonora, Baja California Sur, Coahuila, Chihuahua, Edo. De Méx., Jalisco y Tamaulipas. Podemos observar que el VA/L de las MiPyME es superior en Nacional, Nuevo León y Distrito Federal, mientras que en la GE el resto de los estados presenta un valor más alto.

Tabla 3: Comparación de la ventaja Salarial entre la MiPyME, GE y el Total General (2009).

\begin{tabular}{|l|c|c|c|}
\hline \multicolumn{4}{|c}{ Ventaja por salario } \\
\hline Entidad & MiPyME & Grande & Total General \\
\hline Nacional & 0.99 & 0.98 & 0.99 \\
\hline Nuevo león & 13.96 & 2.20 & 1.12 \\
\hline Distrito federal & 0.44 & 0.48 & 0.45 \\
\hline Baja california & 0.36 & 0.37 & 0.36 \\
\hline Querétaro & 36.65 & 1.37 & 1.11 \\
\hline Sonora & 10.06 & 0.89 & 1.02 \\
\hline Baja california sur & 0.38 & 0.32 & 0.37 \\
\hline Coahuila & 0.33 & 0.35 & 0.33 \\
\hline Chihuahua & 0.33 & 0.33 & 0.33 \\
\hline México & 0.28 & 0.36 & 0.29 \\
\hline Jalisco & 0.34 & 0.27 & 0.33 \\
\hline Tamaulipas & 9.61 & 0.35 & 0.98 \\
\hline
\end{tabular}

Fuente: Elaboración propia con datos del Censo Económico 2009, INEGI. 


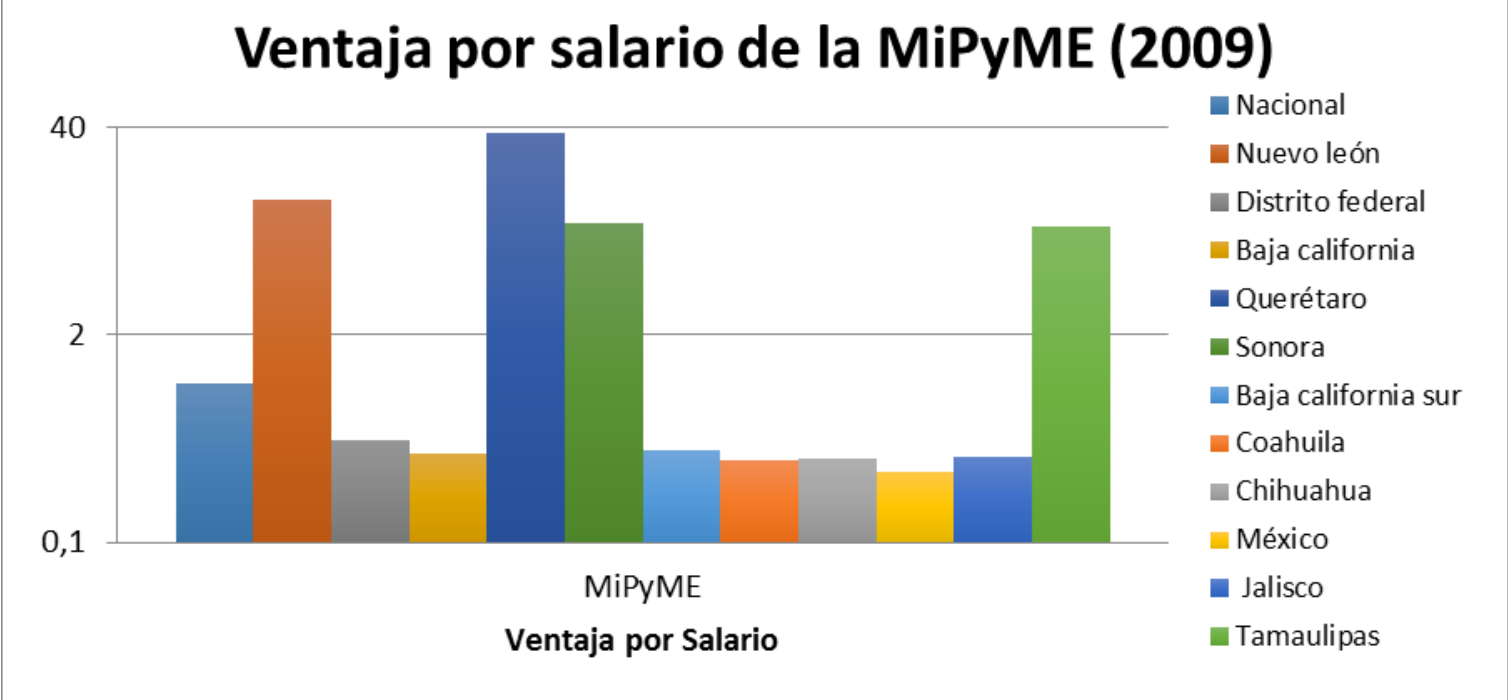

Figura 3: Ventaja por salario de la MiPyME (2009).

Fuente: Elaboración propia con datos del Censo Económico 2009, INEGI.

La tabla 3 y la figura 3 exponen la comparación de la Ventaja Salarial de la MiPyME. El estado de Querétaro muestra una mayor remuneración salarial seguido del estado de Nuevo León, Sonora, Tamaulipas, Nacional, D.F., Baja California Sur, Baja California, Jalisco, Coahuila y Chihuahua se mantienen igual y finalmente Edo. de México.

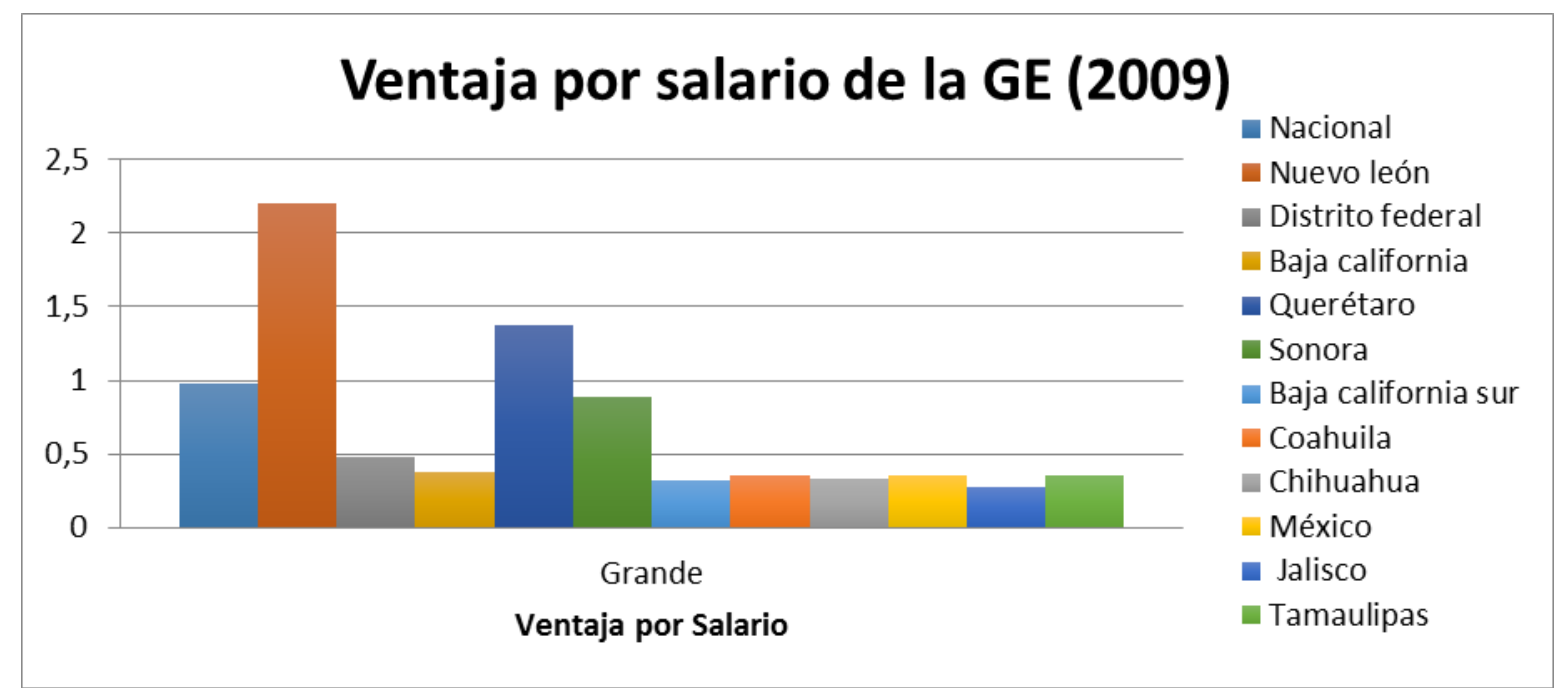

Figura 4: Ventaja por salario de la GE (2009).

Fuente: Elaboración propia con datos del Censo Económico 2009, INEGI.

La figura 4 evidencia la comparación de la Ventaja Salarial de la GE. El estado de Nuevo León, muestra una mayor remuneración salarial seguida de Querétaro, Nacional, Sonora, D.F., Baja California, México, Coahuila y Tamaulipas se mantienen igual, Chihuahua, Baja California Sur y finalmente Jalisco. 


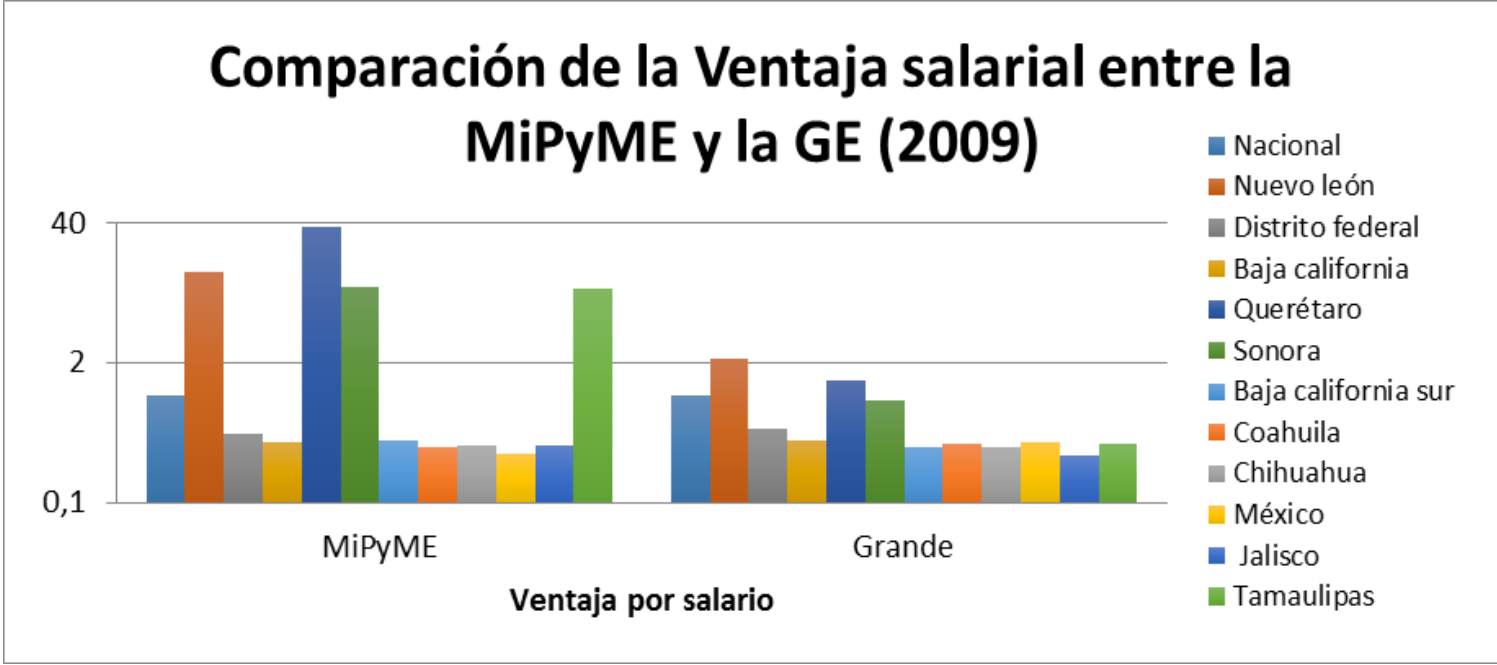

Figura 5: Comparación de la Ventaja salarial entre la MiPyME y la GE (2009).

Fuente: Elaboración propia con datos del Censo Económico 2009, INEGI.

La figura 5 expone la comparación de la Ventaja Salarial entre la MiPyME y GE. La GE en Nuevo León muestra una mayor remuneración salarial, seguido de Querétaro, Nacional, Sonora, D.F., Baja California, México, Coahuila y Tamaulipas se mantienen igual, Chihuahua, Baja California Sur y, finalmente, Jalisco, mientras que en la MiPyME el primer lugar lo obtiene el estado de Querétaro seguido de Nuevo León, Sonora, Tamaulipas, Nacional, D.F., Baja California Sur, Baja California, Jalisco, Coahuila y Chihuahua se mantienen igual y, finalmente, Edo. de México.

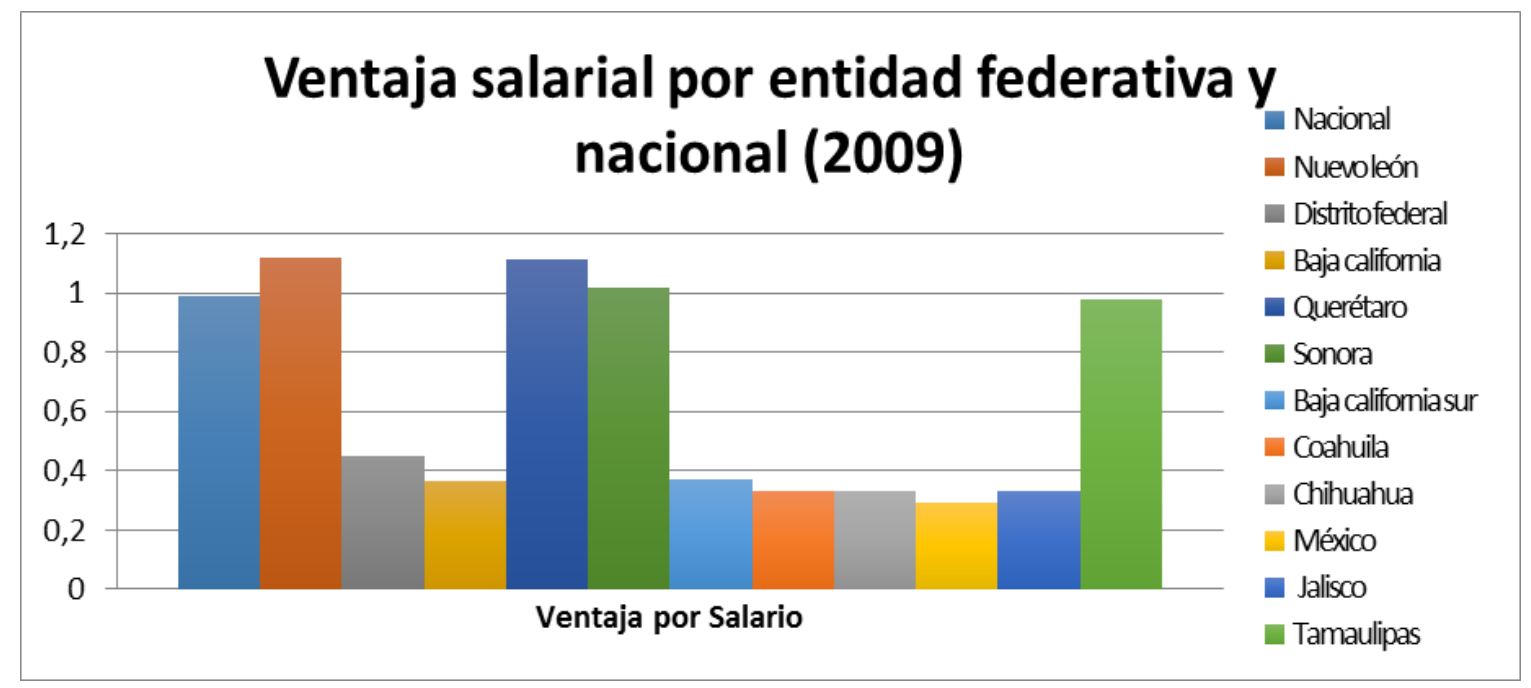

Figura 6: Ventaja salarial por entidad federativa y nacional (2009).

Fuente: Elaboración propia con datos del Censo Económico 2009, INEGI.

La figura 6 presenta la Ventaja por Salario a nivel nacional y de Nuevo León, D.F. Baja California, Querétaro, Sonora, Baja California Sur, Coahuila, Chihuahua, México, Jalisco y Tamaulipas. Como se muestra en la figura el Estado de Nuevo León, presenta mayores niveles salariales, seguido de Querétaro, Sonora, Nacional, Tamaulipas, D.F., Baja California Sur, Baja California, Jalisco, Chihuahua y Coahuila se mantienen con el mismo nivel salarial competitivo y, finalmente, Edo. de México. 


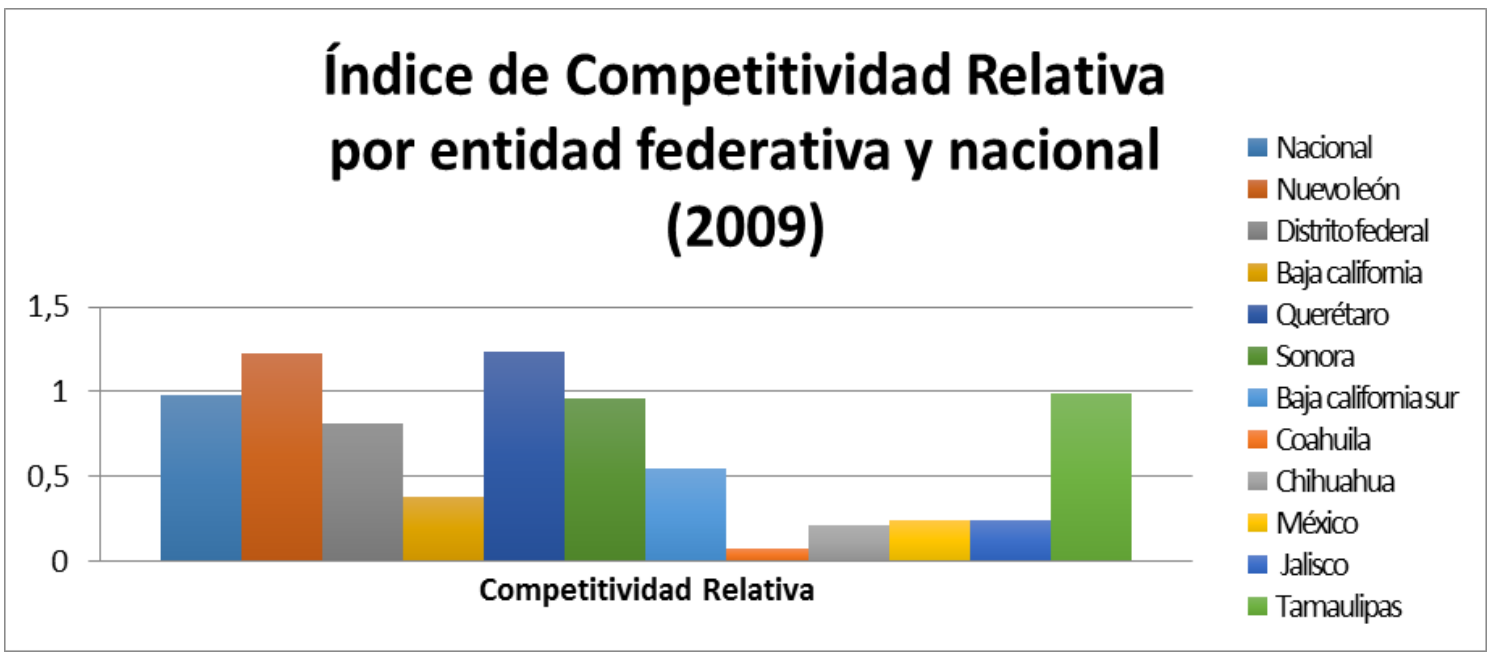

Figura 7: Índice de Competitividad Relativa por entidad federativa y nacional (2009).

Fuente: Elaboración propia con datos del Censo Económico 2009, INEGI.

En la figura 7 se muestra el índice de Competitividad relativa, este índice se obtiene a través de los datos de ventaja por productividad y ventaja por salario. Querétaro es el estado con mayor competitividad relativa, seguido de Nuevo León, Tamaulipas, Nacional, Sonora, D.F., Baja California Sur, Baja California, Jalisco, Edo. De México, Chihuahua, y finalmente Coahuila.

Tabla 4: Comparación de Índices de ventaja por productividad-salario y Competitividad relativa de la micro empresa (2009).

\begin{tabular}{|l|c|c|c|}
\hline \multicolumn{4}{|c}{ Micro } \\
\hline Entidad & $\begin{array}{c}\text { Ventaja por } \\
\text { productividad }\end{array}$ & $\begin{array}{c}\text { Ventaja por } \\
\text { salario }\end{array}$ & $\begin{array}{c}\text { Competitividad } \\
\text { relativa }\end{array}$ \\
\hline Nuevo león & 1.26 & 36.27 & 36.52 \\
\hline Distrito federal & 1.78 & 0.35 & 1.13 \\
\hline Baja california & 0.94 & 0.32 & 0.26 \\
\hline Querétaro & 1.25 & 61.20 & 61.45 \\
\hline Sonora & 1.06 & 20.41 & 20.47 \\
\hline Baja california & 1.06 & 0.32 & 0.38 \\
\hline sur & 0.75 & 0.30 & 0.06 \\
\hline Coahuila & 0.90 & 0.30 & 0.20 \\
\hline Chihuahua & 0.84 & 0.22 & 0.06 \\
\hline México & 0.98 & 0.27 & 0.25 \\
\hline Jalisco & 0.96 & 15.78 & 15.74 \\
\hline Tamaulipas & & & \\
\hline
\end{tabular}

Fuente: Elaboración propia con datos del Censo Económico 2009, INEGI. 


\section{Índices de ventaja por productividad-salario y Competitividad relativa de la Micro Empresa}

(2009)

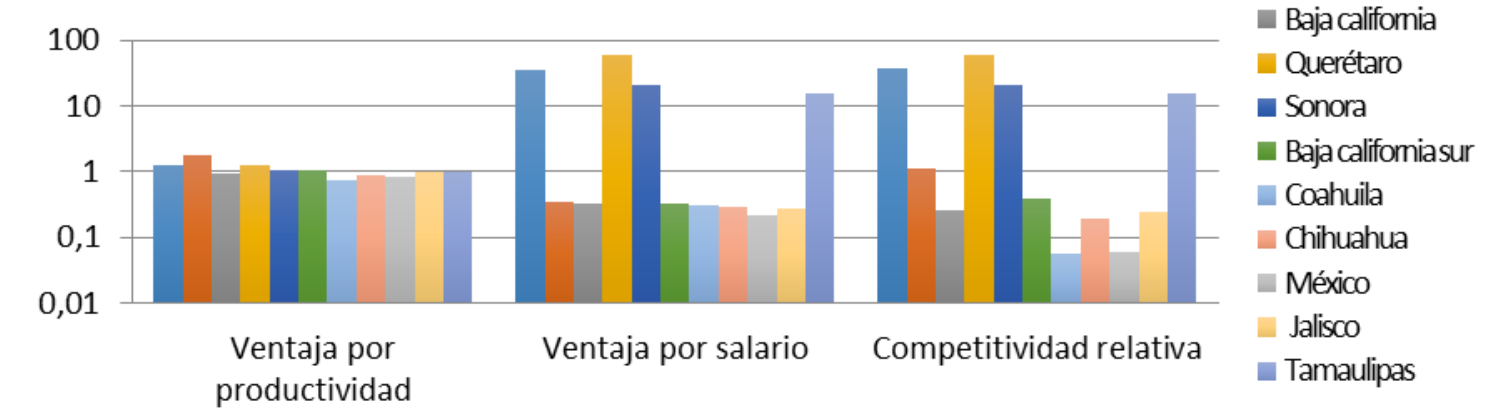

Figura 8: Índices de ventaja por productividad-salario y competitividad relativa de la Micro Empresa (2009).

Fuente: Elaboración propia con datos del Censo Económico 2009, INEGI.

La tabla 4 y la figura 8 exhiben los índices elaborados de la ventaja por productividad, la ventaja por salario y la competitividad relativa de la micro empresa para Nuevo León, D.F., Baja California, Querétaro, Sonora, Baja California Sur, Coahuila, Chihuahua, Edo. De Méx., Jalisco y Tamaulipas. Podemos observar que Querétaro presenta índices superiores a nivel salarial y competitivo. Mientras que en la ventaja por productividad el D.F., es el más competitivo.

Tabla 5: Comparación de Índices de ventaja por productividad-salario y Competitividad relativa de la PyME (2009).

\begin{tabular}{|l|c|c|c|}
\hline \multicolumn{4}{|c}{ PyME } \\
\hline Entidad & $\begin{array}{c}\text { Ventaja por } \\
\text { productividad }\end{array}$ & $\begin{array}{c}\text { Ventaja } \\
\text { por salario }\end{array}$ & $\begin{array}{c}\text { Competitividad } \\
\text { relativa }\end{array}$ \\
\hline Nuevo león & 1.13 & 13.96 & 14.09 \\
\hline Distrito federal & 1.43 & 0.44 & 0.87 \\
\hline Baja california & 0.99 & 0.36 & 0.35 \\
\hline Querétaro & 1.19 & 36.65 & 36.85 \\
\hline Sonora & 0.99 & 10.06 & 10.05 \\
\hline Baja california & 1.19 & 0.38 & 0.57 \\
\hline sur & & & \\
\hline Coahuila & 0.75 & 0.33 & 0.08 \\
\hline Chihuahua & 0.96 & 0.33 & 0.29 \\
\hline México & 0.94 & 0.28 & 0.21 \\
\hline Jalisco & 0.96 & 0.34 & 0.30 \\
\hline Tamaulipas & 1.06 & 9.61 & 9.66 \\
\hline
\end{tabular}

Fuente: Elaboración propia con datos del Censo Económico 2009, INEGI. 
Figura 9: Índices de ventaja por productividad-salario y Competitividad relativa de la PyME (2009).

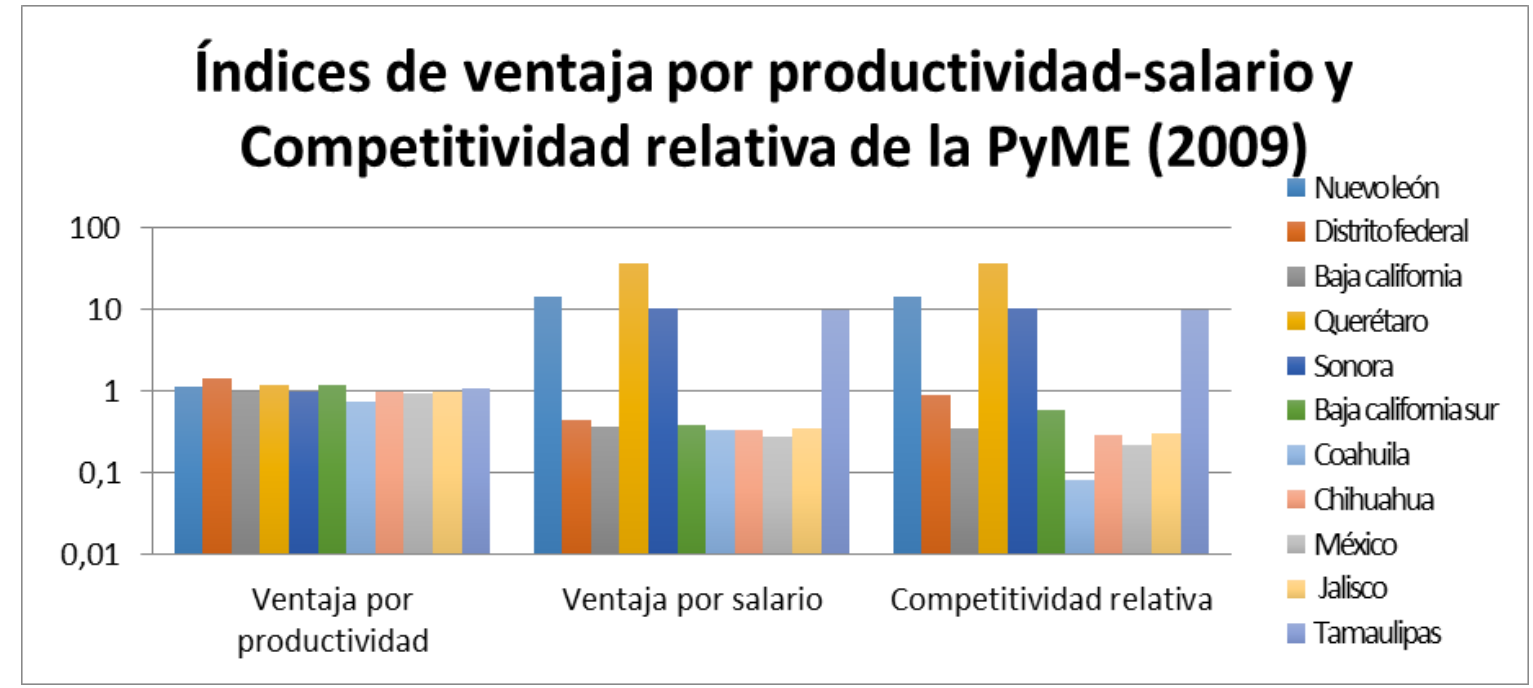

Figura 9: Índices de ventaja por productividad-salario y competitividad relativa de la PyMe (2009).

Fuente: Elaboración propia con datos del Censo Económico 2009, INEGI.

La tabla 5 y la figura 9 presentan los índices elaborados de la ventaja por productividad, la ventaja por salario y la competitividad relativa de la PyME para Nuevo León, D.F., Baja California, Querétaro, Sonora, Baja California Sur, Coahuila, Chihuahua, Edo. De Méx., Jalisco y Tamaulipas. El D.F., presenta un mayor nivel en la ventaja por productividad, mientras que Querétaro sobresale por mucho en la ventaja salarial, así mismo muestra un comportamiento más elevado en la competitividad relativa.

Tabla 6: Comparación de Índices de ventaja por productividad-salario y Competitividad relativa de la Gran Empresa (2009).

\begin{tabular}{|l|c|c|c|}
\hline \multicolumn{4}{|c|}{ Grande } \\
\hline Entidad & $\begin{array}{c}\text { Ventaja por } \\
\text { productividad }\end{array}$ & $\begin{array}{c}\text { Ventaja } \\
\text { por salario }\end{array}$ & $\begin{array}{c}\text { Competitividad } \\
\text { relativa }\end{array}$ \\
\hline Nuevo león & 0.97 & 2.20 & 2.17 \\
\hline Distrito federal & 1.15 & 0.48 & 0.63 \\
\hline Baja california & 1.15 & 0.37 & 0.52 \\
\hline Querétaro & 0.76 & 1.37 & 1.13 \\
\hline Sonora & 0.69 & 0.89 & 0.57 \\
\hline Baja california & 0.99 & 0.32 & 0.32 \\
\hline sur & & & \\
\hline Coahuila & 0.68 & 0.35 & 0.04 \\
\hline Chihuahua & 0.50 & 0.33 & -0.17 \\
\hline México & 1.01 & 0.36 & 0.37 \\
\hline Jalisco & 0.73 & 0.27 & 0.01 \\
\hline Tamaulipas & 0.79 & 0.35 & 0.14 \\
\hline
\end{tabular}

Fuente: Elaboración propia con datos del Censo Económico 2009, INEGI. 


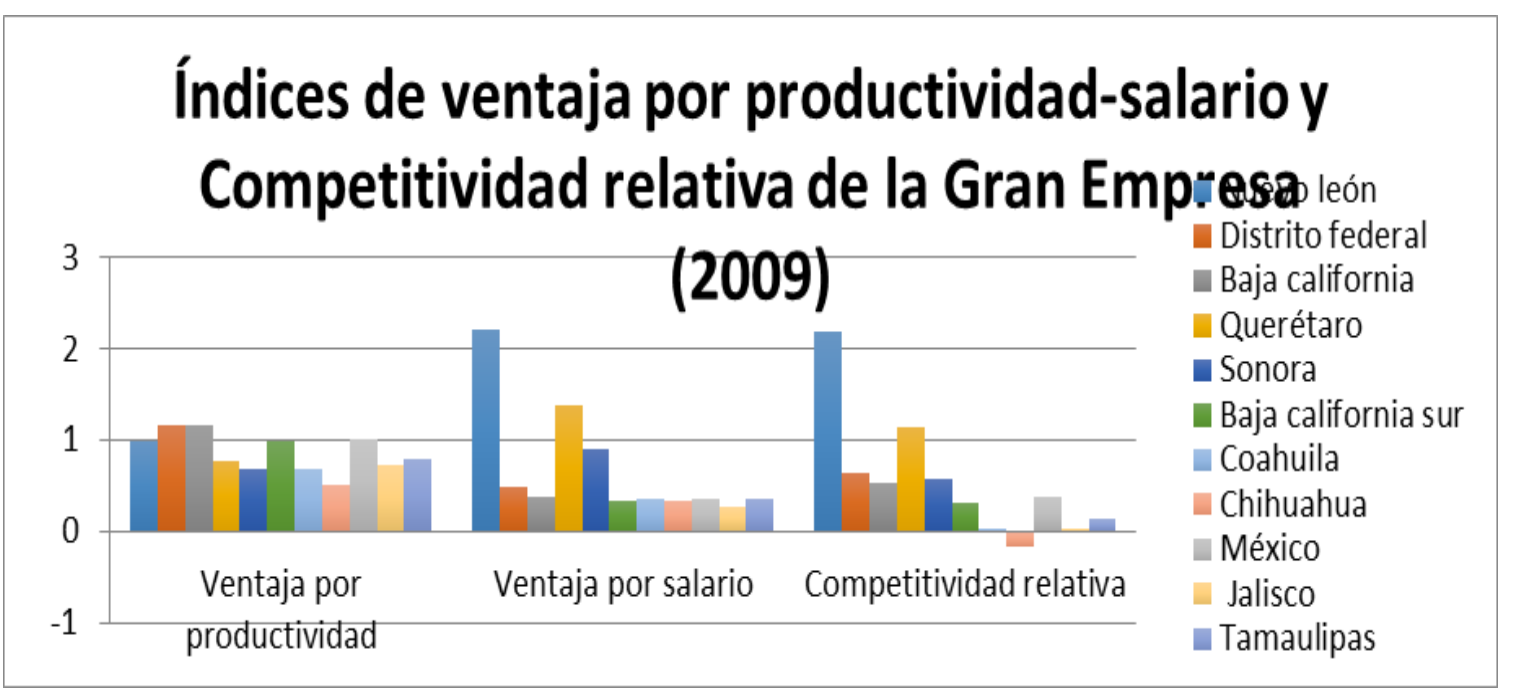

Figura 10: Índices de ventaja por productividad-salario y Competitividad relativa de la Gran Empresa (2009).

Fuente: Elaboración propia con datos del Censo Económico 2009, INEGI.

La tabla 6 y la figura 10 muestran los índices elaborados de la ventaja por productividad, la ventaja por salario y la competitividad relativa de la gran empresa para Nuevo León, D.F., Baja California, Querétaro, Sonora, Baja California Sur, Coahuila, Chihuahua, Edo. De Méx., Jalisco y Tamaulipas. En ésta figura resalta el índice de ventaja salarial y competitividad relativa de Nuevo León siendo de 2.20 y 2.17 respectivamente, con un índice muy elevado en comparación del resto de las entidades.

En la figura 11 se presenta el índice de Competitividad relativa, este índice se obtiene a través de los datos de ventaja por productividad y ventaja por salario. Michoacán es el estado con mayor competitividad relevada, seguido de, Tabasco, Guerrero, Nayarit, Quintana Roo, San Luis Potosí, Veracruz, Yucatán, Sinaloa, Morelos, Puebla, Tlaxcala, Zacatecas, Oaxaca, Campeche, Guanajuato, Hidalgo, Chiapas, Aguascalientes, colima y finalmente Durango. El estado de Michoacán, Tabasco, Nayarit y Quintana Roo están debajo de 3.16 y el resto de los estados son mayores al -0.15 .

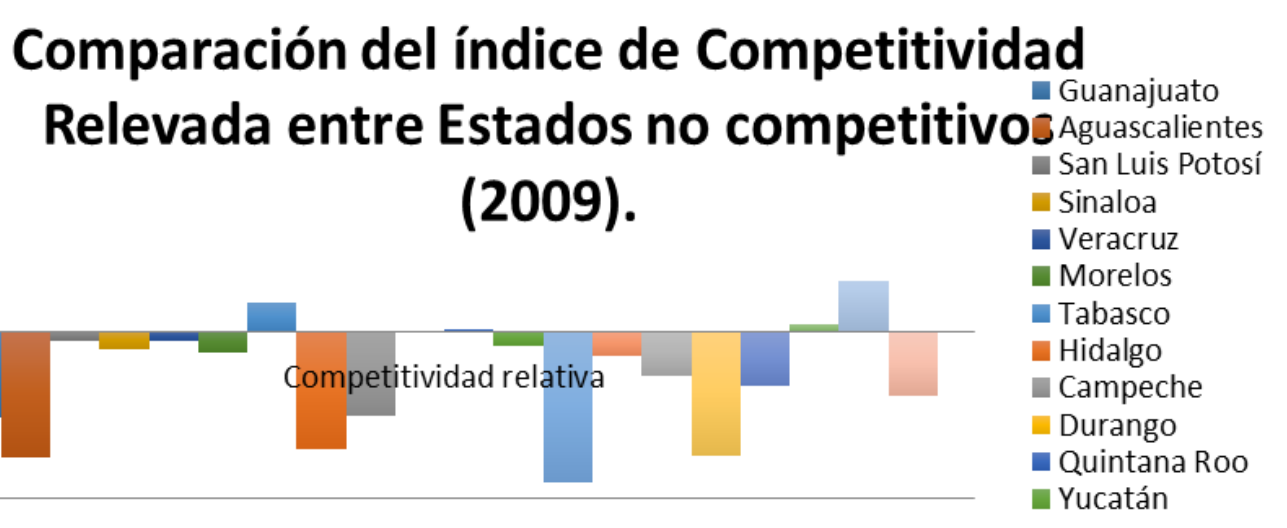

Figura 11: Comparación del índice de Competitividad Relevada entre Estados no competitivos (2009).

Fuente: Elaboración propia con datos del Censo Económico 2009, INEGI. 
Tabla 7: Comparación de Valor Agregado entre Personal Remunerado (2009).

\begin{tabular}{|l|c|c|c|}
\hline \multicolumn{4}{|c|}{ Promedio de VA/L } \\
\hline Entidad & MiPyME & Grande & Total General \\
\hline Nacional & 17999.62 & 950.45 & 13796.80 \\
\hline Guanajuato & 231.57 & 541.79 & 282.42 \\
\hline Aguascalientes & 218.88 & 343.05 & 231.07 \\
\hline San Luis Potosí & 264.81 & 428.93 & 285.20 \\
\hline Sinaloa & 249.66 & 260.04 & 251.28 \\
\hline Veracruz & 108.52 & 924.57 & 245.26 \\
\hline Morelos & 225.22 & 397.13 & 244.66 \\
\hline Tabasco & 608.18 & 1369.73 & 715.06 \\
\hline Hidalgo & 206.34 & 796.18 & 276.56 \\
\hline Campeche & 162.02 & 2279.67 & 437.69 \\
\hline Durango & 182.64 & 418.81 & 214.04 \\
\hline Quintana Roo & 252.87 & 358.47 & 268.13 \\
\hline Yucatán & 229.21 & 221.35 & 228.06 \\
\hline Colima & 237.96 & 250.81 & 239.07 \\
\hline Puebla & 204.48 & 381.03 & 232.10 \\
\hline Tlaxcala & 169.80 & 687.63 & 195.15 \\
\hline Chiapas & 233.89 & 1548.09 & 390.34 \\
\hline Zacatecas & 108.96 & 1018.96 & 179.42 \\
\hline Nayarit & 401.74 & 535.86 & 413.47 \\
\hline Michoacán & 355.97 & 4232.74 & 823.86 \\
\hline Oaxaca & 103.94 & 70.10 & 101.89 \\
\hline Guerrero & 543.62 & 316.79 & 519.46 \\
\hline
\end{tabular}

Fuente: Elaboración propia con datos del Censo Económico 2009, INEGI.

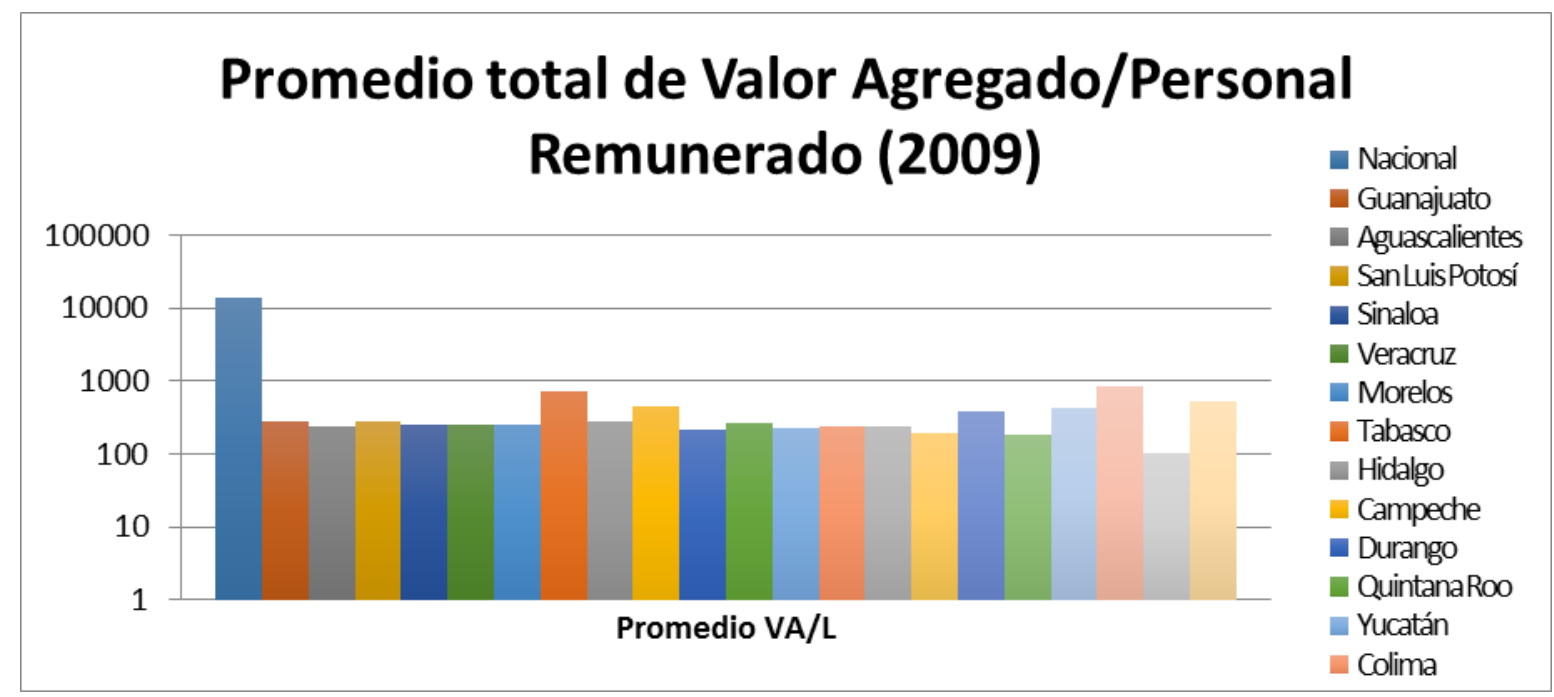

Figura 12: Promedio total de Valor Agregado/Personal Remunerado (2009). Fuente: Elaboración propia con datos del Censo Económico 2009, INEGI. 
La tabla 7 y la figura 12 muestran el comportamiento del Valor Agregado/Personal Remunerado del promedio Nacional, Aguascalientes, Campeche, Chiapas, Colima, Durango, Guanajuato, Guerrero, Hidalgo, Michoacán, Morelos, Nayarit, Oaxaca, Puebla, Quintana Roo, San Luis Potosí, Sinaloa, Tabasco, Tlaxcala, Veracruz, Yucatán y Zacatecas. El promedio Nacional presenta un mayor nivel competitivo de Valor Agregado, le sigue el promedio Michoacán, Tabasco, Guerrero, Campeche, Nayarit, Chiapas, San Luis Potosí, Hidalgo, Quintana Roo, Sinaloa, Veracruz, Morelos, Colima, Puebla, Aguascalientes, Yucatán, Durango, Tlaxcala, Zacatecas y, Finalmente, el estado de Oaxaca.

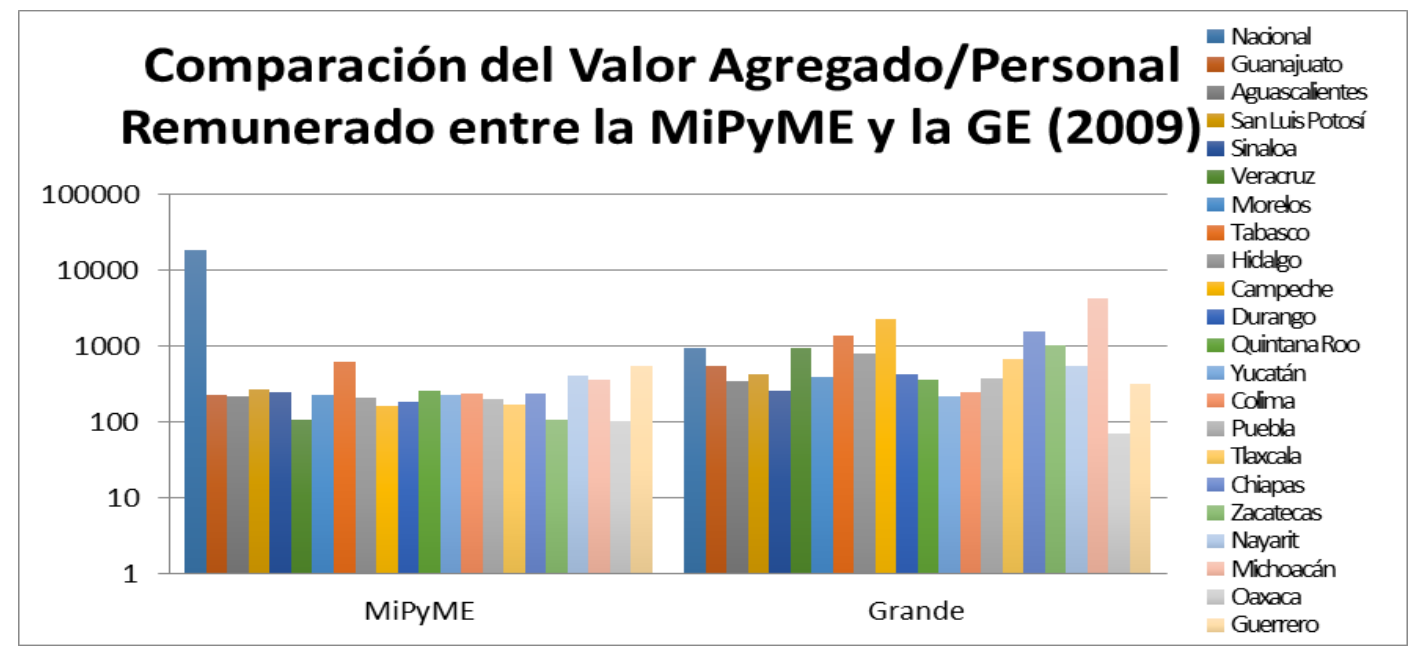

Figura 13: Comparación del Valor Agregado/Personal Remunerado entre la MiPyME y la GE (2009). Fuente: Elaboración propia con datos del Censo Económico 2009, INEGI.

La figura 13 evidencia la comparación del valor agregado/ personal remunerado entre la MiPyME y la Gran Empresa del promedio Nacional, Aguascalientes, Campeche, Chiapas, Colima, Durango, Guanajuato, Guerrero, Hidalgo, Michoacán, Morelos, Nayarit, Oaxaca, Puebla, Quintana Roo, San Luis Potosí, Sinaloa, Tabasco, Tlaxcala, Veracruz, Yucatán y Zacatecas. Podemos observar que el VA/L de la Gran Empresa es mayor al de la PyME a excepción del promedio Nacional.

En la figura 14 se muestra el comportamiento del Total de Remuneraciones/Personal Remunerado a nivel nacional, Aguascalientes, Campeche, Chiapas, Colima, Durango, Guanajuato, Guerrero, Hidalgo, Michoacán, Morelos, Nayarit, Oaxaca, Puebla, Quintana Roo, San Luis Potosí, Sinaloa, Tabasco, Tlaxcala, Veracruz, Yucatán y Zacatecas. Como se muestra en la figura, Tabasco presenta una mayor competitividad en Ventaja Salarial, seguido del promedio Nacional, Guanajuato, Campeche, Durango, Quintana Roo, San Luis Potosí, Veracruz, Puebla, Aguascalientes, Zacatecas, Morelos, Sinaloa, Hidalgo, Colima, Michoacán, Guerrero, Nayarit, Chiapas, Yucatán, Tlaxcala y, finalmente, Oaxaca. 


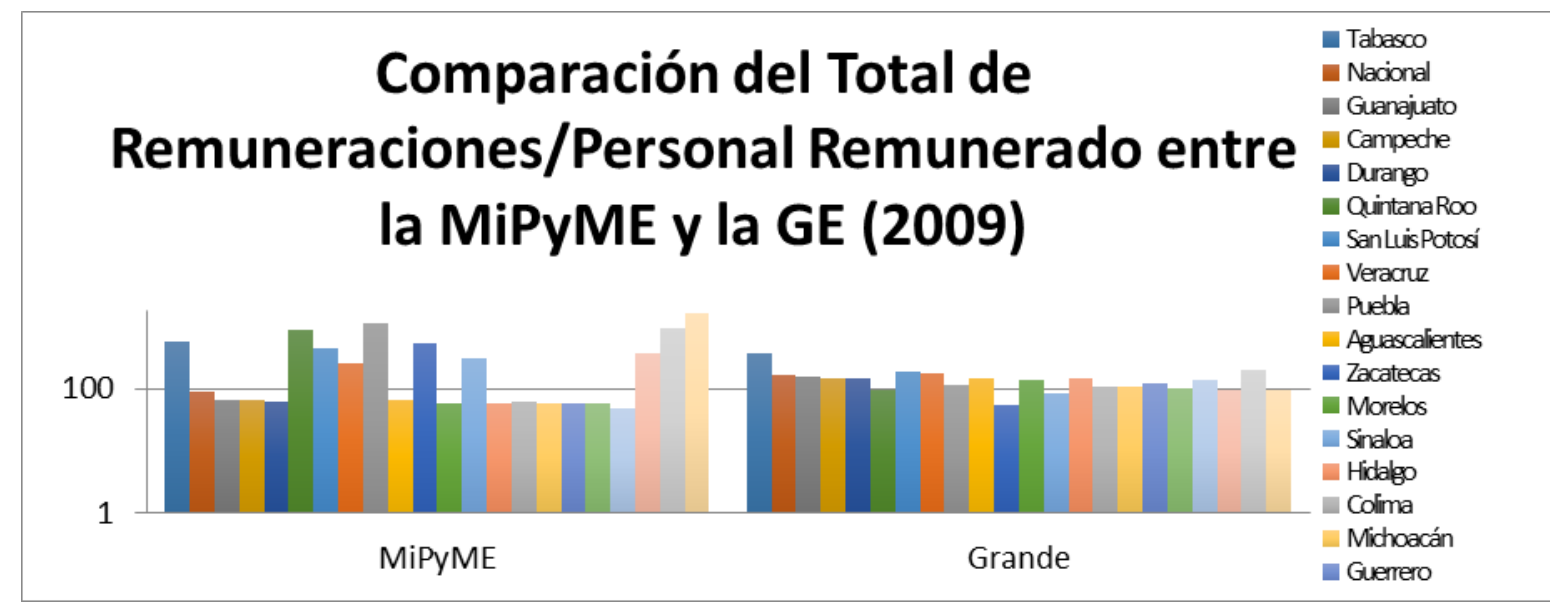

Figura 14: Comparación del Total de Remuneraciones/Personal Remunerado entre la MiPyME y la GE (2009).

Fuente: Elaboración propia con datos del censo económico 2009, INEGI.

Esta figura presenta la comparación total de Remuneraciones/Personal Remunerado de la MiPyME y la Gran Empresa. La MiPyME muestra salarios mayores en los estados de Quintana Roo, San Luis Potosí, Puebla, Zacates, Sinaloa, Yucatán, Tlaxcala y Oaxaca que en la GE. Mientras que el resto de los estados se mantienen con menor remuneración salarial que los de la GE.

Tabla 8: Comparación de la ventaja Salarial entre la MiPyME, GE y el Total General (2009).

\begin{tabular}{|l|c|c|c|}
\hline \multicolumn{4}{|c|}{ Ventaja por Salario } \\
\hline Entidad & MiPyME & Grande & Total General \\
\hline Nacional & 0.99 & 0.98 & 0.99 \\
\hline Guanajuato & 0.29 & 0.37 & 0.30 \\
\hline Aguascalientes & 0.29 & 0.42 & 0.31 \\
\hline San Luis Potosí & 11.41 & 1.13 & 0.95 \\
\hline Sinaloa & 7.06 & 0.72 & 0.91 \\
\hline Veracruz & 5.21 & 2.35 & 0.84 \\
\hline Morelos & 0.86 & 1.05 & 0.88 \\
\hline Tabasco & 8.20 & 5.52 & 1.11 \\
\hline Hidalgo & 0.28 & 0.33 & 0.28 \\
\hline Campeche & 0.28 & 0.39 & 0.29 \\
\hline Durango & 0.26 & 0.33 & 0.27 \\
\hline Quintana Roo & 21.41 & 0.56 & 1.01 \\
\hline Yucatán & 7.56 & 0.93 & 0.80 \\
\hline Colima & 0.29 & 0.26 & 0.29 \\
\hline Puebla & 30.34 & 0.93 & 0.84 \\
\hline Tlaxcala & 20.05 & 1.98 & 0.77 \\
\hline Chiapas & 0.23 & 0.30 & 0.24 \\
\hline Zacatecas & 10.13 & 0.53 & 0.89 \\
\hline Nayarit & 0.86 & 0.82 & 0.85 \\
\hline
\end{tabular}




\begin{tabular}{|l|c|c|c|}
\hline Michoacán & 0.27 & 0.30 & 0.28 \\
\hline Oaxaca & 26.84 & 0.73 & 0.74 \\
\hline Guerrero & 0.27 & 0.38 & 0.29 \\
\hline
\end{tabular}

Fuente: Elaboración propia con datos del Censo Económico 2009, INEGI.

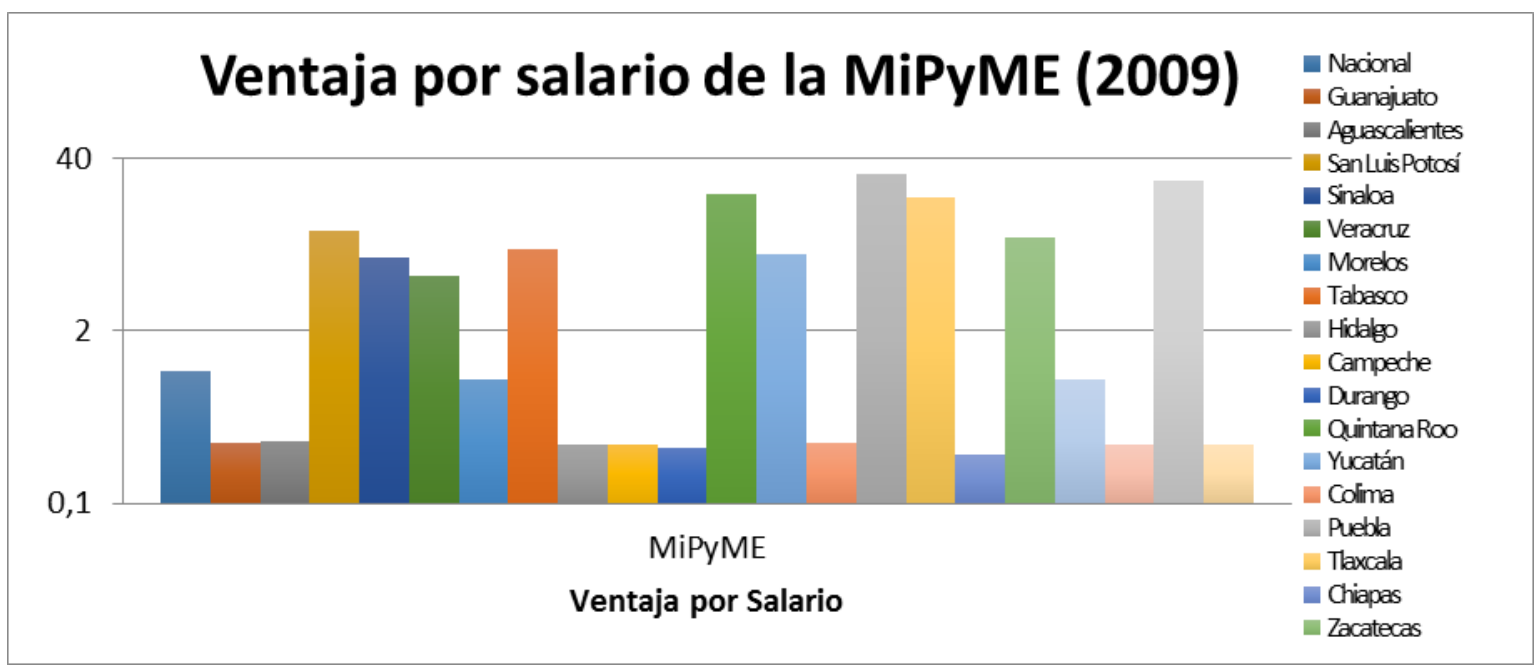

Figura 15: Ventaja por salario de la MiPyME (2009).

Fuente: Elaboración propia con datos del censo económico 2009, INEGI.

La tabla 8 y la figura 15 muestran la comparación de la Ventaja Salarial de la MiPyME. El estado de Puebla muestra una mayor remuneración salarial seguido del estado de Oaxaca, Quintana Roo, Tlaxcala, San Luis Potosí, Zacatecas, Tabasco, Yucatán, Sinaloa, Veracruz, Promedio Nacional, Morelos, Nayarit, Aguascalientes, Guanajuato, Colima, Campeche, Hidalgo, Guerrero, Michoacán, Durango y Chiapas.

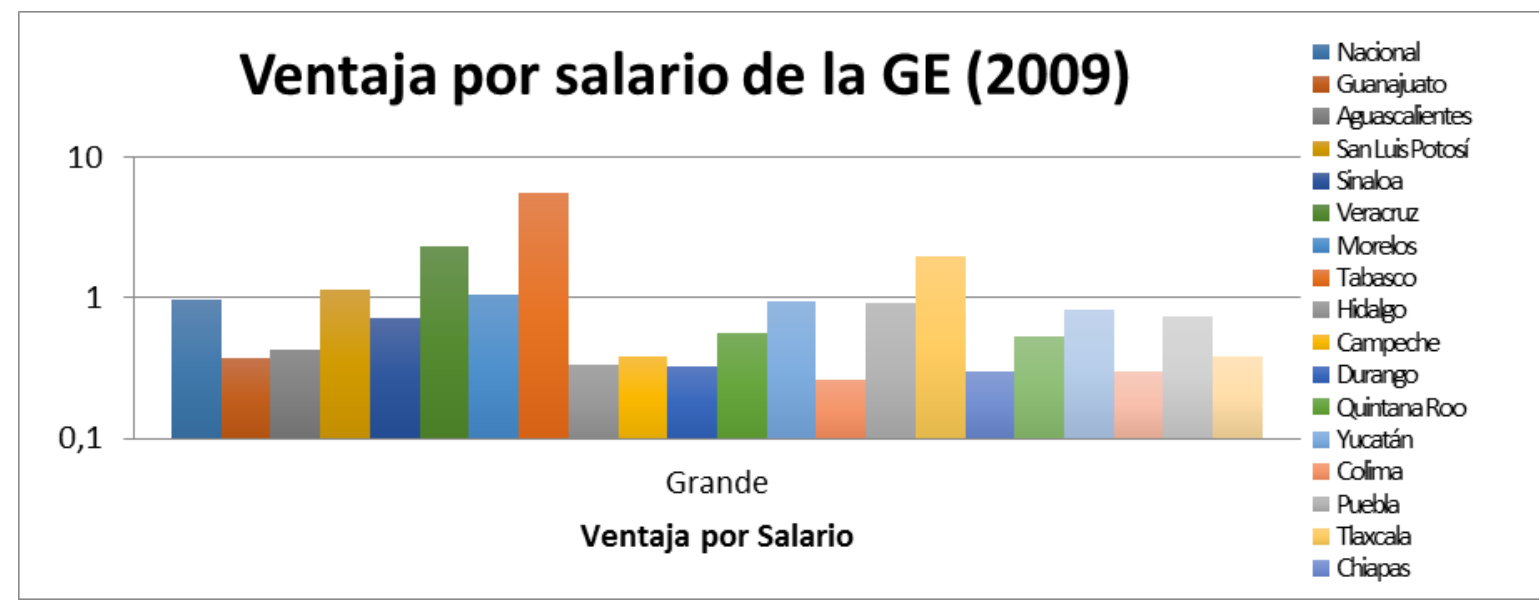

Figura 16: Ventaja por salario de la GE (2009).

Fuente: Elaboración propia con datos del censo económico 2009, INEGI.

La figura 16 muestra la comparación de la Ventaja Salarial de la GE. El estado de Tabasco muestra una mayor remuneración salarial seguido de Veracruz, Tlaxcala, San Luis Potosí, Morelos, Media Nacional, Yucatán, Puebla, Nayarit, Oaxaca, Sinaloa, Quintana Roo, Zacatecas, Aguascalientes, Campeche, Guerrero, Guanajuato, Hidalgo, Durango, Chiapas, Michoacán y, finalmente, Colima. 


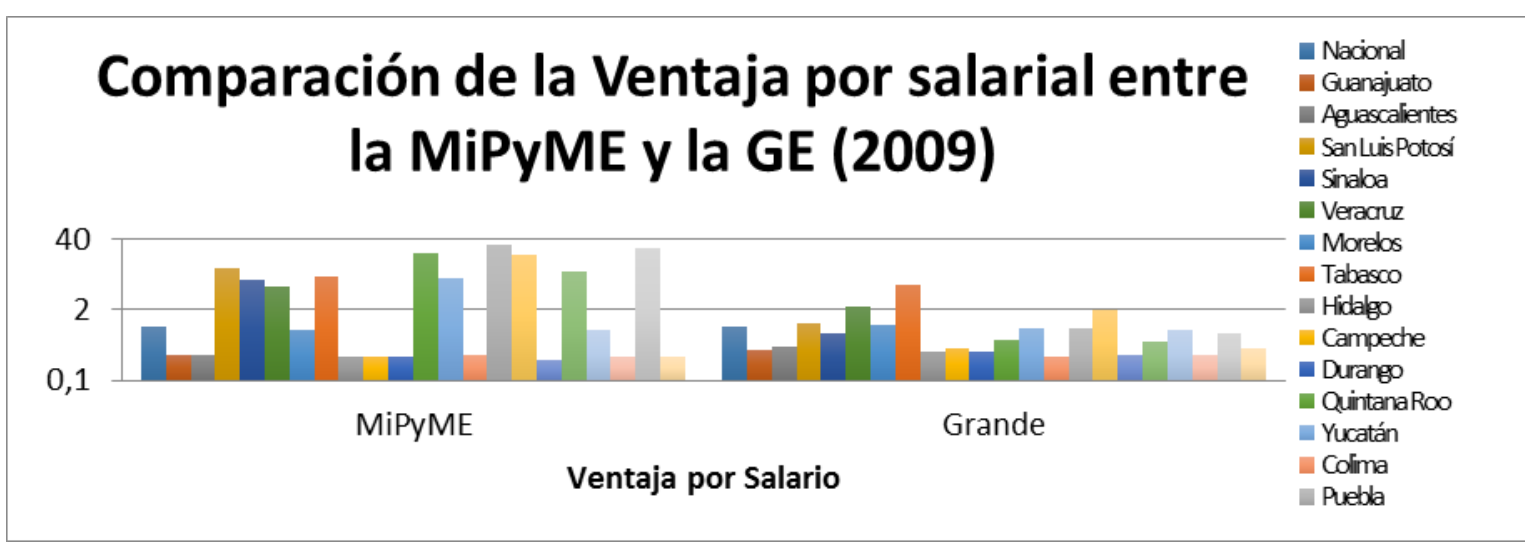

Figura 17: Comparación de la Ventaja por salarial entre la MiPyME y la GE (2009). Fuente: Elaboración propia con datos del censo económico 2009, INEGI.

En la figura 17 se muestra la comparación de la Ventaja Salarial entre la MiPyME y GE. Podemos observar que el estado con mayor remuneración salarial de la MiPyME es el estado de Puebla y en la GE Tabasco es el estado mejor remunerado. La MiPyME cuenta con estados mejor remunerados que la GE. Las remuneraciones son menores a $30.3 \mathrm{y}$ mayores a 0.2. Mientras que en la GE la remuneración es mayor a 0.2 y menor a 5.5

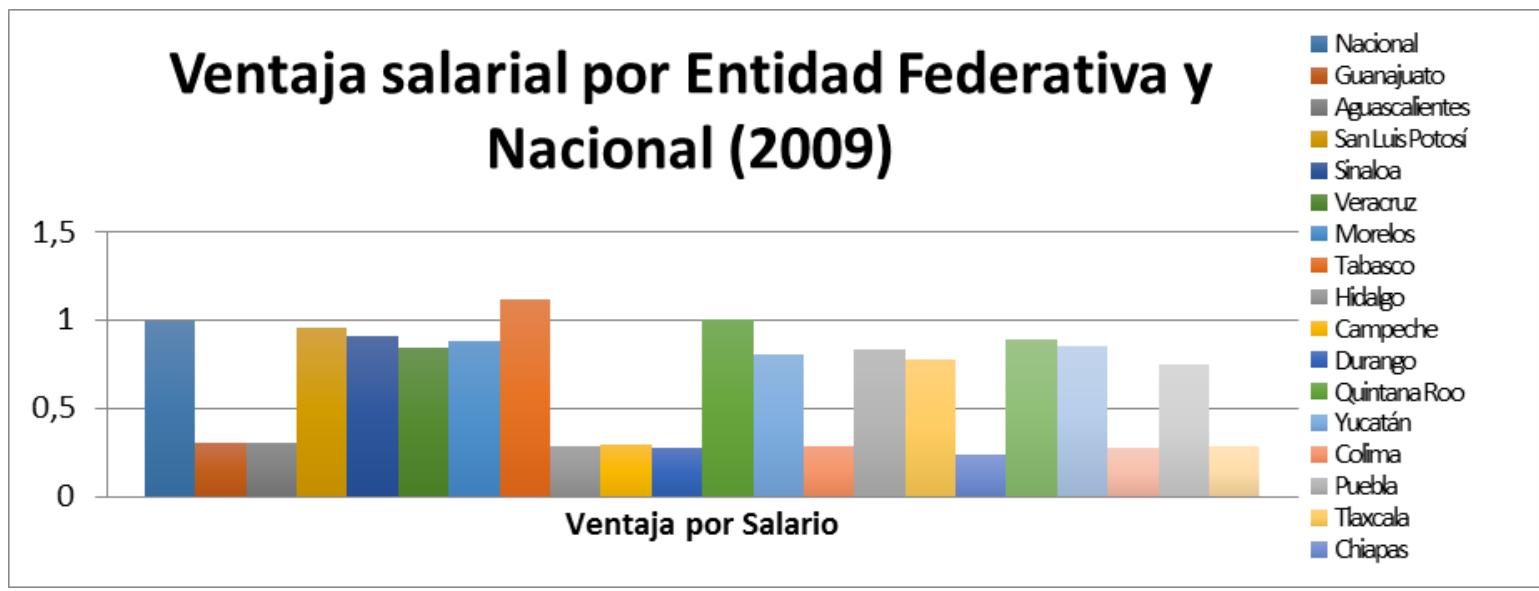

Figura 18. Ventaja salarial por Entidad Federativa y Nacional (2009).

Fuente: Elaboración propia con datos del Censo Económico 2009, INEGI.

En la figura 18 se presenta la ventaja por Salario del promedio Nacional, Aguascalientes, Campeche, Chiapas, Colima, Durango, Guanajuato, Guerrero, Hidalgo, Michoacán, Morelos, Nayarit, Oaxaca, Puebla, Quintana Roo, San Luis Potosí, Sinaloa, Tabasco, Tlaxcala, Veracruz, Yucatán y Zacatecas. Como se muestra en la figura, el Estado de Tabasco, seguido del estado de Quintana Roo, Promedio Nacional, San Luis Potosí Sinaloa, Zacatecas, Morelos, Nayarit, Veracruz, Puebla, Yucatán, Tlaxcala, Oaxaca, Aguascalientes, Guanajuato, Campeche, Colima, Guerrero, Hidalgo, Michoacán, Durango y, finalmente, Chiapas.

En la tabla 9 y la figura 19 se presenta el índice de Competitividad relativa, este índice se obtiene a través de los datos de ventaja por productividad y ventaja por salario. Michoacán es el estado con mayor competitividad relativa, seguido de Tabasco, Guerreo, Nayarit, Quintana Roo, Promedio Nacional, San Luis Potosí, Veracruz, Yucatán, Sinaloa, Morelos, 
Puebla, Tlaxcala, Zacatecas, Oaxaca, Campeche, Hidalgo, Chiapas, Aguascalientes, Guanajuato, Colima y, finalmente, Durango.

Tabla 9: Comparación de Índices de ventaja por productividad-salario y Competitividad relativa de la micro empresa (2009).

\begin{tabular}{|c|c|c|c|}
\hline \multicolumn{4}{|c|}{ Micro } \\
\hline Entidad & $\begin{array}{l}\text { Ventaja por } \\
\text { productividad }\end{array}$ & $\begin{array}{l}\text { Ventaja } \\
\text { por salario }\end{array}$ & $\begin{array}{l}\text { Competitividad } \\
\text { relativa }\end{array}$ \\
\hline Guanajuato & 0.86 & 0.27 & 0.14 \\
\hline Aguascalientes & 0.98 & 0.25 & 0.23 \\
\hline San Luis Potosí & 0.80 & 23.88 & 23.68 \\
\hline Sinaloa & 1.06 & 15.62 & 15.68 \\
\hline Veracruz & 0.89 & 11.05 & 10.94 \\
\hline Morelos & 0.78 & 0.88 & 0.66 \\
\hline Tabasco & 0.95 & 9.00 & 8.95 \\
\hline Hidalgo & 0.69 & 0.22 & -0.09 \\
\hline Campeche & 0.70 & 0.23 & -0.07 \\
\hline Durango & 0.25 & 0.24 & -0.51 \\
\hline Quintana Roo & 0.93 & 15.59 & 15.52 \\
\hline Yucatán & 1.54 & 14.54 & 15.08 \\
\hline Colima & 0.90 & 0.28 & 0.18 \\
\hline Puebla & 1.00 & 59.64 & 59.64 \\
\hline Tlaxcala & 0.63 & 33.98 & 33.61 \\
\hline Chiapas & 0.92 & 0.19 & 0.11 \\
\hline Zacatecas & 0.75 & 18.14 & 17.89 \\
\hline Nayarit & 2.22 & 0.93 & 2.15 \\
\hline Michoacán & 0.90 & 0.24 & 0.14 \\
\hline Oaxaca & 0.73 & 18.71 & 18.44 \\
\hline Guerrero & 1.16 & 0.23 & 0.39 \\
\hline
\end{tabular}

Fuente: Elaboración propia con datos del Censo Económico 2009, INEGI.

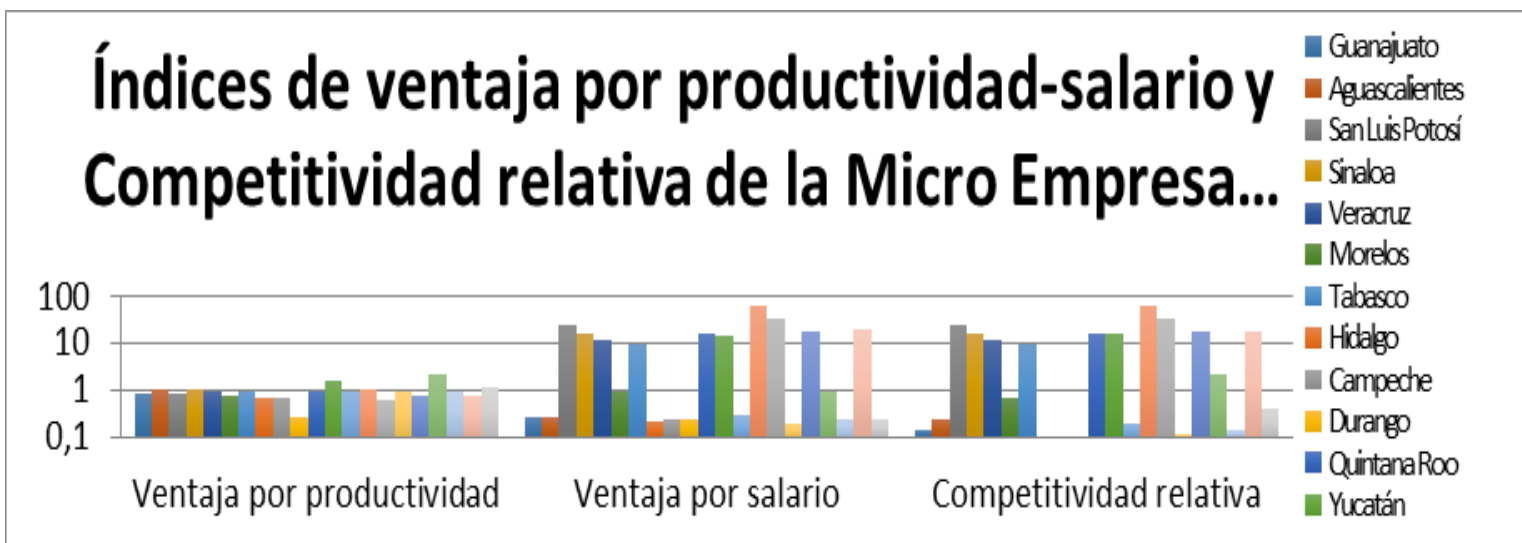

Figura 19: Índices de ventaja por productividad-salario y Competitividad relativa de la Micro Empresa (2009).

Fuente: Elaboración propia con datos del Censo Económico 2009, INEGI. 
La tabla 9 y la figura 19 muestran los índices elaborados de la ventaja por productividad, la ventaja por salario y la competitividad relativa de la micro empresa para Aguascalientes, Campeche, Chiapas, Colima, Durango, Guanajuato, Guerrero, Hidalgo, Michoacán, Morelos, Nayarit, Oaxaca, Puebla, Quintana Roo, San Luis Potosí, Sinaloa, Tabasco, Tlaxcala, Veracruz, Yucatán y Zacatecas. Podemos observar que Puebla presenta índices superiores a nivel salarial y competitivo, mientras que en la ventaja por productividad Nayarit es el más competitivo.

Tabla 10: Comparación de Índices de ventaja por productividad-salario y Competitividad relativa de la MiPyME (2009).

\begin{tabular}{|l|c|c|c|}
\hline \multicolumn{4}{|c}{ MiPyME } \\
\hline Entidad & $\begin{array}{c}\text { Ventaja por } \\
\text { productividad }\end{array}$ & $\begin{array}{c}\text { Ventaja } \\
\text { por salario }\end{array}$ & $\begin{array}{c}\text { Competitividad } \\
\text { relativa }\end{array}$ \\
\hline Guanajuato & 0.80 & 0.29 & 0.09 \\
\hline Aguascalientes & 0.87 & 0.29 & 0.16 \\
\hline San Luis Potosí & 0.96 & 11.41 & 11.37 \\
\hline Sinaloa & 0.94 & 7.06 & 7.00 \\
\hline Veracruz & 1.03 & 5.21 & 5.24 \\
\hline Morelos & 0.84 & 0.86 & 0.70 \\
\hline Tabasco & 1.40 & 8.20 & 8.61 \\
\hline Hidalgo & 0.89 & 0.28 & 0.17 \\
\hline Campeche & 0.91 & 0.28 & 0.19 \\
\hline Durango & 0.56 & 0.26 & -0.18 \\
\hline Quintana Roo & 1.03 & 21.41 & 21.43 \\
\hline Yucatán & 1.09 & 7.56 & 7.66 \\
\hline Colima & 0.87 & 0.29 & 0.16 \\
\hline Puebla & 0.89 & 30.34 & 30.23 \\
\hline Tlaxcala & 0.72 & 20.05 & 19.77 \\
\hline Chiapas & 0.88 & 0.23 & 0.12 \\
\hline Zacatecas & 0.56 & 10.13 & 9.69 \\
\hline Nayarit & 1.26 & 0.86 & 1.12 \\
\hline Michoacán & 1.55 & 0.27 & 0.82 \\
\hline Oaxaca & 0.69 & 26.84 & 26.53 \\
\hline Guerrero & 2.30 & 0.27 & 1.57 \\
\hline Fuente: & & & 0.19 \\
\hline
\end{tabular}

Fuente: Elaboración propia con datos del Censo Económico 2009, INEGI. 


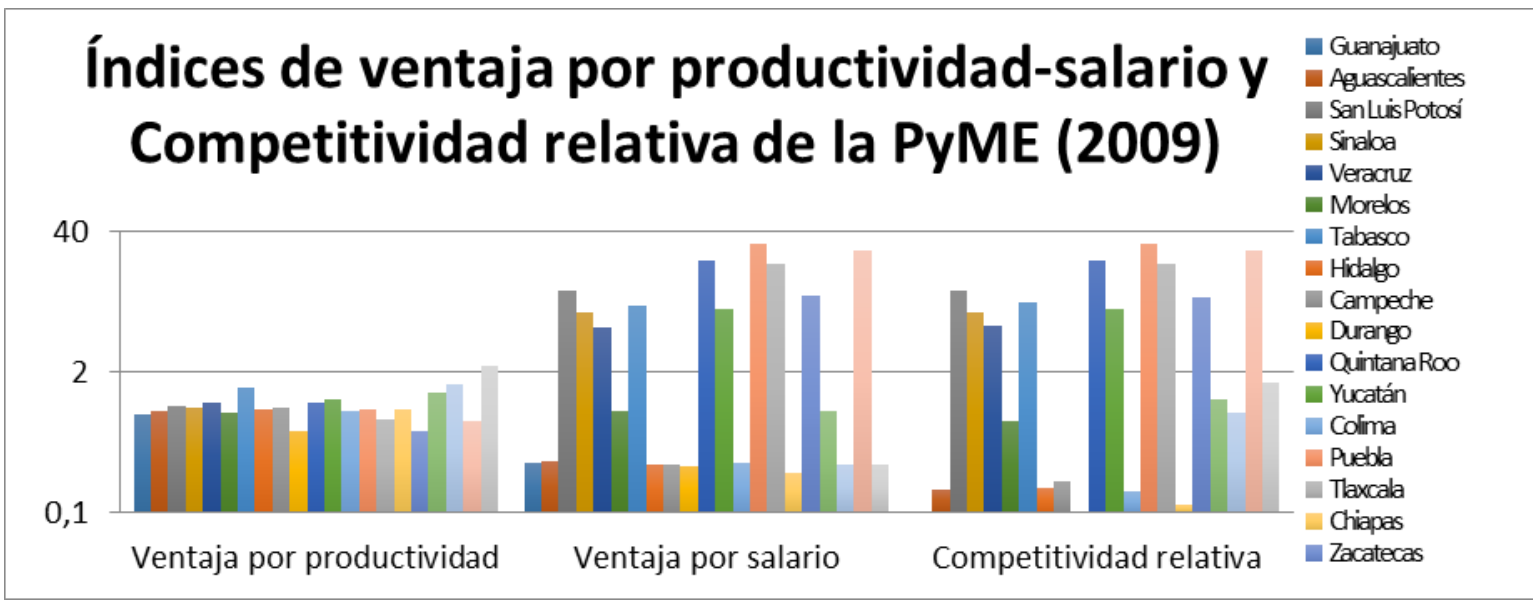

Figura 20: Índices de ventaja por productividad-salario y Competitividad relativa de la PyME (2009). Fuente: Elaboración propia con datos del Censo Económico 2009, INEGI.

La tabla 10 y la figura 20 exponen los índices elaborados de la ventaja por productividad, la ventaja por salario y la competitividad relativa de la PyME para Aguascalientes, Campeche, Chiapas, Colima, Durango, Guanajuato, Guerrero, Hidalgo, Michoacán, Morelos, Nayarit, Oaxaca, Puebla, Quintana Roo, San Luis Potosí, Sinaloa, Tabasco, Tlaxcala, Veracruz, Yucatán y Zacatecas. El estado de Guerrero presenta un mayor nivel en la ventaja por productividad, mientras que Puebla sobresale por mucho en la ventaja salarial, así mismo muestra un comportamiento más elevado en la competitividad relativa.

Tabla 11: Comparación de Índices de ventaja por productividad-salario y Competitividad relativa de la Gran Empresa (2009).

\begin{tabular}{|l|c|c|c|}
\hline & $\begin{array}{c}|c| \\
\text { Ventaja por } \\
\text { productividad }\end{array}$ & $\begin{array}{c}\text { Ventaja por } \\
\text { salario }\end{array}$ & $\begin{array}{c}\text { Competitividad } \\
\text { relativa }\end{array}$ \\
\hline Guanajuato & 0.95 & 0.37 & 0.33 \\
\hline Aguascalientes & 0.86 & 0.42 & 0.28 \\
\hline San Luis Potosí & 0.79 & 1.13 & 0.93 \\
\hline Sinaloa & 0.49 & 0.72 & 0.21 \\
\hline Veracruz & 1.09 & 2.35 & 2.44 \\
\hline Morelos & 1.07 & 1.05 & 1.12 \\
\hline Tabasco & 1.33 & 5.52 & 5.85 \\
\hline Hidalgo & 1.10 & 0.33 & 0.43 \\
\hline Campeche & 1.71 & 0.39 & 1.10 \\
\hline Durango & 0.65 & 0.33 & -0.02 \\
\hline Quintana Roo & 0.88 & 0.56 & 0.45 \\
\hline Yucatán & 0.55 & 0.93 & 0.48 \\
\hline Colima & 0.53 & 0.26 & -0.20 \\
\hline Puebla & 0.87 & 0.93 & 0.80 \\
\hline Tlaxcala & 1.55 & 1.98 & 2.53 \\
\hline Chiapas & 1.37 & 0.30 & 0.67 \\
\hline Zacatecas & 0.88 & 0.53 & 0.41 \\
\hline & & & \\
\hline
\end{tabular}




\begin{tabular}{|l|c|c|c|}
\hline Nayarit & 1.29 & 0.82 & 1.11 \\
\hline Michoacán & 20.90 & 0.30 & 20.20 \\
\hline Oaxaca & 0.40 & 0.73 & 0.14 \\
\hline Guerrero & 0.80 & 0.38 & 0.18 \\
\hline
\end{tabular}

Fuente: Elaboración propia con datos del Censo Económico 2009, INEGI.

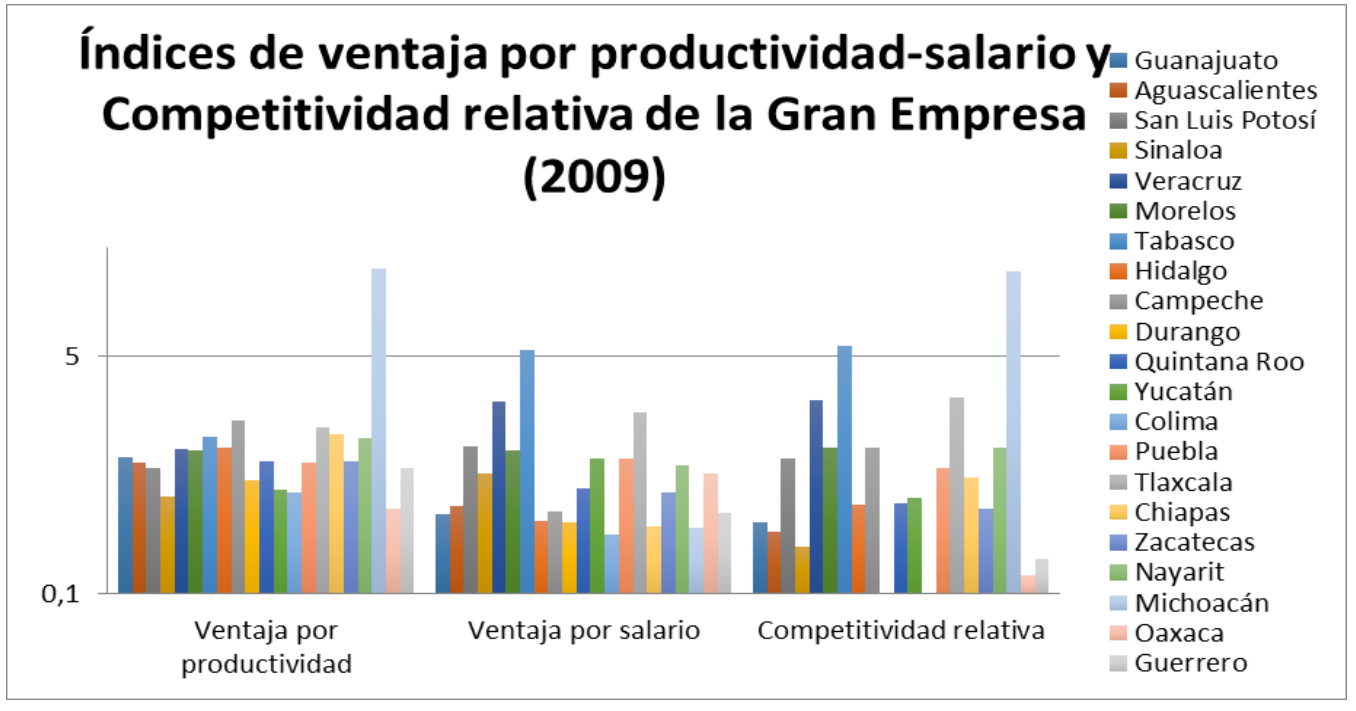

Figura 21: Índices de ventaja por productividad-salario y Competitividad relativa de la Gran Empresa (2009).

Fuente: Elaboración propia con datos del Censo Económico 2009, INEGI.

La figura 21 presenta los índices elaborados de la ventaja por productividad, la ventaja por salario y la competitividad relativa de la GE para Aguascalientes, Campeche, Chiapas, Colima, Durango, Guanajuato, Guerrero, Hidalgo, Michoacán, Morelos, Nayarit, Oaxaca, Puebla, Quintana Roo, San Luis Potosí, Sinaloa, Tabasco, Tlaxcala, Veracruz, Yucatán y Zacatecas. En esta figura resalta el índice de ventaja por productividad y competitividad relativa de Michoacán siendo de 20.8 y 20.1 respectivamente, con un índice muy elevado en comparación del resto de las entidades, mientas que en la ventaja salarial el estado mejor remunerado es Tabasco.

Tabla 12: Índices de ventaja por productividad-salario y Competitividad relativa de Guanajuato (2009).

\begin{tabular}{|l|c|c|c|}
\hline Guanajuato & $\begin{array}{c}\text { Ventaja por } \\
\text { productividad }\end{array}$ & $\begin{array}{c}\text { Ventaja por } \\
\text { salario }\end{array}$ & $\begin{array}{c}\text { Competitividad } \\
\text { relativa }\end{array}$ \\
\hline Micro & 0.863540519 & 0.272550159 & 0.136090678 \\
\hline PyME & 0.800179017 & 0.289495156 & 0.089674173 \\
\hline Grande & 0.950981386 & 0.374265123 & 0.325246509 \\
\hline
\end{tabular}

Fuente: Elaboración propia con datos del Censo Económico 2009, INEGI. 
Tabla 13: Ordenamiento de Competitividad, Productividad y Salarios: Correlaciones de rango del Total de los Estados.

\begin{tabular}{|c|c|c|c|c|c|c|}
\hline Estado & $\mathrm{C}^{* *}$ & $\begin{array}{l}\text { ORDEN } \\
\text { DE C** }\end{array}$ & (VA/L)* & $\begin{array}{c}\text { ORDEN } \\
\text { DE } \\
\text { (VA/L)* }\end{array}$ & $(W / L)^{*}$ & $\begin{array}{l}\text { ORDEN } \\
\text { DE (W/L) }\end{array}$ \\
\hline Querétaro & 31.09 & 1 & 1.12 & 7 & 30.97 & 1 \\
\hline Puebla & 25.63 & 2 & 0.89 & 21 & 25.74 & 2 \\
\hline Oaxaca & 24.93 & 3 & 0.67 & 30 & 25.26 & 3 \\
\hline Tlaxcala & 18.93 & 4 & 0.76 & 28 & 19.17 & 4 \\
\hline Quintana roo & 18.40 & 5 & 1.01 & 14 & 18.39 & 5 \\
\hline Nuevo león & 11.71 & 6 & 1.10 & 8 & 11.61 & 6 \\
\hline San Luis potosí & 10.07 & 7 & 0.94 & 18 & 10.13 & 7 \\
\hline Zacatecas & 8.97 & 8 & 0.59 & 31 & 9.39 & 8 \\
\hline Sonora & 8.39 & 9 & 0.94 & 17 & 8.45 & 9 \\
\hline Tamaulipas & 8.23 & 10 & 1.02 & 11 & 8.22 & 10 \\
\hline Tabasco & 8.22 & 11 & 1.39 & 3 & 7.83 & 11 \\
\hline Yucatán & 6.61 & 12 & 1.02 & 12 & 6.59 & 12 \\
\hline Sinaloa & 5.94 & 13 & 0.87 & 23 & 6.07 & 13 \\
\hline Veracruz & 4.77 & 14 & 1.04 & 9 & 4.73 & 14 \\
\hline Michoacán & 3.16 & 15 & 3.89 & 1 & 0.28 & 30 \\
\hline Guerrero & 1.42 & 16 & 2.14 & 2 & 0.29 & 28 \\
\hline Nayarit & 1.12 & 17 & 1.27 & 5 & 0.85 & 16 \\
\hline Distrito federal & 0.81 & 18 & 1.36 & 4 & 0.45 & 17 \\
\hline Morelos & 0.74 & 19 & 0.86 & 25 & 0.88 & 15 \\
\hline Baja california sur & 0.54 & 20 & 1.17 & 6 & 0.37 & 18 \\
\hline Baja california & 0.37 & 21 & 1.01 & 13 & 0.36 & 19 \\
\hline Campeche & 0.31 & 22 & 1.02 & 10 & 0.29 & 25 \\
\hline Jalisco & 0.24 & 23 & 0.91 & 20 & 0.33 & 22 \\
\hline México & 0.24 & 24 & 0.95 & 15 & 0.29 & 26 \\
\hline Colima & 0.21 & 25 & 0.87 & 22 & 0.33 & 20 \\
\hline Hidalgo & 0.20 & 26 & 0.92 & 19 & 0.28 & 29 \\
\hline Coahuila & 0.18 & 27 & 0.94 & 16 & 0.24 & 32 \\
\hline Aguascalientes & 0.18 & 28 & 0.87 & 24 & 0.31 & 23 \\
\hline Guanajuato & 0.13 & 29 & 0.82 & 27 & 0.30 & 24 \\
\hline Chihuahua & 0.12 & 30 & 0.84 & 26 & 0.29 & 27 \\
\hline Chiapas & 0.07 & 31 & 0.74 & 29 & 0.33 & 21 \\
\hline Durango & -0.16 & 32 & 0.57 & 32 & 0.27 & 31 \\
\hline \multicolumn{7}{|c|}{ CORRELACIÓN DE RANGO(SPERMAN) } \\
\hline \multicolumn{3}{|c|}{ C** y (VA/L)*: 0.256267} & \multicolumn{2}{|c|}{$\begin{array}{l}(V A / L) * Y(W / L)^{*}: \\
0.013703\end{array}$} & \multicolumn{2}{|l|}{$\begin{array}{l}C^{* *} \quad y \\
0.891544\end{array}$} \\
\hline
\end{tabular}

Fuente: Elaboración propia con datos del Censo Económico 2009, INEGI. 
Como se expone en la tabla 13, se observa la posición en que se colocó cada estado de la República Mexicana en la comparación de la Competitividad, Productividad y Salarios del Total General, donde 1 significa que es el estado más competitivo y 32 el menos competitivo. Así mismo, se calculó las correlaciones de rango del Total General de los Estados; en el caso de $C^{* *}$ y $(\mathrm{VA} / \mathrm{L}) *$ : 0.25626; y $(\mathrm{VA} / \mathrm{L}) *$ Y $(\mathrm{W} / \mathrm{L}) *$ : 0.013703; como el valor se acerca a 0 quiere decir que no hay correlación lineal en el caso de $C^{* *}$ y $(\mathrm{W} / \mathrm{L})^{*}$ : 0.891544 existe una correlación positiva.

Tabla 14: Ordenamiento de Competitividad, Productividad y Salarios: Correlaciones de rango de la MiPyME de los Estados.

\begin{tabular}{|l|c|c|c|c|c|c|}
\hline Estado & C** & $\begin{array}{c}\text { ORDEN } \\
\text { DE C** }\end{array}$ & (VA/L) & $\begin{array}{c}\text { ORDEN DE } \\
\text { (VA/L)* }\end{array}$ & $\begin{array}{c}\text { (W/L)* } \\
\text { (W/L) }\end{array}$ \\
\hline Querétaro & 36.85 & 1 & 1.19 & 6 & 36.65 & 1 \\
\hline Puebla & 30.23 & 2 & 0.89 & 22 & 30.34 & 2 \\
\hline Oaxaca & 26.53 & 3 & 0.69 & 30 & 26.84 & 3 \\
\hline Quintana roo & 21.43 & 4 & 1.03 & 12 & 21.41 & 4 \\
\hline Tlaxcala & 19.77 & 5 & 0.72 & 29 & 20.05 & 5 \\
\hline Nuevo león & 14.09 & 6 & 1.13 & 8 & 13.96 & 6 \\
\hline San Luis potosín & 11.37 & 7 & 0.96 & 15 & 11.41 & 7 \\
\hline Sonora & 10.05 & 8 & 0.99 & 13 & 10.06 & 9 \\
\hline Zacatecas & 9.69 & 9 & 0.56 & 31 & 10.13 & 8 \\
\hline Tamaulipas & 9.66 & 10 & 1.06 & 10 & 9.61 & 10 \\
\hline Tabasco & 8.61 & 11 & 1.40 & 4 & 8.20 & 11 \\
\hline Yucatán & 7.66 & 12 & 1.09 & 9 & 7.56 & 12 \\
\hline Sinaloa & 7.00 & 13 & 0.94 & 18 & 7.06 & 13 \\
\hline Veracruz & 5.24 & 14 & 1.03 & 11 & 5.21 & 14 \\
\hline Guerrero & 1.57 & 15 & 2.30 & 1 & 0.27 & 29 \\
\hline Nayarit & 1.12 & 16 & 1.26 & 5 & 0.86 & 16 \\
\hline Distrito federal & 0.87 & 17 & 1.43 & 3 & 0.44 & 17 \\
\hline Michoacán & 0.82 & 18 & 1.55 & 2 & 0.27 & 30 \\
\hline Morelos & 0.70 & 19 & 0.84 & 26 & 0.86 & 15 \\
\hline Baja california sur & 0.57 & 20 & 1.19 & 7 & 0.38 & 18 \\
\hline Baja california & 0.35 & 21 & 0.99 & 14 & 0.36 & 19 \\
\hline Jalisco & 0.30 & 22 & 0.96 & 17 & 0.34 & 20 \\
\hline Colima & 0.29 & 23 & 0.96 & 16 & 0.33 & 21 \\
\hline México & 0.21 & 24 & 0.94 & 19 & 0.28 & 27 \\
\hline Campeche & 0.19 & 25 & 0.91 & 20 & 0.28 & 26 \\
\hline Hidalgo & 0.17 & 26 & 0.89 & 21 & 0.28 & 28 \\
\hline Aguascalientes & 0.16 & 27 & 0.87 & 24 & 0.29 & 23 \\
\hline Chihuahua & 0.16 & 28 & 0.87 & 25 & 0.29 & 25 \\
\hline Coahuila & 0.12 & 29 & 0.88 & 23 & 0.23 & 32 \\
\hline Guanajuato & 0.09 & 30 & 0.80 & 27 & 0.29 & 24 \\
\hline Chiapas & 31 & 0.75 & 28 & 0.33 & 22 \\
\hline
\end{tabular}




\begin{tabular}{|c|c|c|c|c|}
\hline $\begin{array}{l}\text { Durango }-0.18 \\
\text { CORRELACIÓN DE RANGO(SPERMAN) } \\
\text { C** v (VA/L)* } 0.313085\end{array}$ & 0.56 & 32 & 0.26 & 31 \\
\hline \multicolumn{5}{|l|}{ CORRELACIÓN DE RANGO(SPERMAN) } \\
\hline C** y (VA/L)*: 0.313085 & $\begin{array}{l}(\mathrm{VA} / \mathrm{L})^{*} \\
0.093332\end{array}$ & Y $\quad(W / L) *$ & $\begin{array}{l}C^{* *} \text { y } \\
0.903075\end{array}$ & $(W / L)^{*}:$ \\
\hline
\end{tabular}

Fuente: Elaboración propia con datos del Censo Económico 2009, INEGI.

Como se aprecia en la tabla 14, se observa la posición en que se colocó cada estado de la República Mexicana en la comparación de la Competitividad, Productividad y Salarios de la MiPyME, donde 1 significa que es el estado más competitivo y 32 el menos competitivo. Así mismo, se calculó las correlaciones de rango del Total General de los Estados; en el caso de $\mathrm{C}^{* *}$ y $(\mathrm{VA} / \mathrm{L}) *$ : 0.313085; y $(\mathrm{VA} / \mathrm{L})^{*} \mathrm{Y}(\mathrm{W} / \mathrm{L})^{*}$ : 0.093332; como el valor se acerca a 0 quiere decir que no hay correlación lineal en el caso de $C^{* *}$ y $(\mathrm{W} / \mathrm{L})^{*}$ : 0.903075 existe una correlación positiva.

Tabla 15: Ordenamiento de Competitividad, Productividad y Salarios: Correlaciones de rango de la Gran Empresa de los Estados.

\begin{tabular}{|c|c|c|c|c|c|c|}
\hline Estado & $C^{* *}$ & $\begin{array}{l}\text { ORDEN DE } \\
\mathrm{C}^{* *}(\mathrm{VA} / \mathrm{L})^{*}\end{array}$ & (VA/L)* & $\begin{array}{c}\text { ORDEN } \\
\text { DE } \\
\text { (VA/L)* }\end{array}$ & $(W / L)^{*}$ & $\begin{array}{c}\text { ORDEN } \\
\text { DE (W/L) }\end{array}$ \\
\hline Nuevo león & 5.85 & 1 & 0.97 & 14 & 2.20 & 3 \\
\hline Quintana roo & 2.53 & 2 & 0.88 & 16 & 0.56 & 14 \\
\hline Oaxaca & 2.44 & 3 & 0.40 & 32 & 0.73 & 12 \\
\hline Veracruz & 2.17 & 4 & 1.09 & 10 & 2.35 & 2 \\
\hline Tabasco & 1.13 & 5 & 1.33 & 5 & 5.52 & 1 \\
\hline Nayarit & 1.12 & 6 & 1.29 & 6 & 0.82 & 11 \\
\hline Guerrero & 1.11 & 7 & 0.80 & 20 & 0.38 & 19 \\
\hline Colima & 1.10 & 8 & 0.53 & 29 & 0.26 & 32 \\
\hline Durango & 0.96 & 9 & 0.65 & 27 & 0.33 & 27 \\
\hline Zacatecas & 0.93 & 10 & 0.88 & 17 & 0.53 & 15 \\
\hline Yucatán & 0.80 & 11 & 0.55 & 28 & 0.93 & 8 \\
\hline Campeche & 0.67 & 12 & 1.71 & 2 & 0.39 & 18 \\
\hline Chihuahua & 0.63 & 13 & 0.50 & 30 & 0.33 & 26 \\
\hline San Luis potosí & 0.57 & 14 & 0.79 & 21 & 1.13 & 6 \\
\hline Guanajuato & 0.52 & 15 & 0.95 & 15 & 0.37 & 20 \\
\hline Puebla & 0.48 & 16 & 0.87 & 18 & 0.93 & 9 \\
\hline Tamaulipas & 0.45 & 17 & 0.79 & 22 & 0.35 & 24 \\
\hline Morelos & 0.43 & 18 & 1.07 & 11 & 1.05 & 7 \\
\hline Querétaro & 0.41 & 19 & 0.76 & 23 & 1.37 & 5 \\
\hline Distrito federal & 0.37 & 20 & 1.15 & 7 & 0.48 & 16 \\
\hline Baja california & 0.33 & 21 & 1.15 & 8 & 0.37 & 21 \\
\hline Chiapas & 0.32 & 22 & 1.37 & 4 & 0.30 & 29 \\
\hline Coahuila & 0.28 & 23 & 0.68 & 26 & 0.35 & 23 \\
\hline Sonora & 0.21 & 24 & 0.69 & 25 & 0.89 & 10 \\
\hline
\end{tabular}




\begin{tabular}{|l|l|l|c|c|c|c|}
\hline Baja california sur & 0.18 & 25 & 0.99 & 13 & 0.32 & 28 \\
\hline Tlaxcala & 0.14 & 26 & 1.55 & 3 & 1.98 & 4 \\
\hline Sinaloa & 0.14 & 27 & 0.49 & 31 & 0.72 & 13 \\
\hline Aguascalientes & 0.04 & 28 & 0.86 & 19 & 0.42 & 17 \\
\hline Michoacán & 0.01 & 29 & 20.90 & 1 & 0.30 & 30 \\
\hline Jalisco & -0.02 & 30 & 0.73 & 24 & 0.27 & 31 \\
\hline México & -0.17 & 31 & 1.01 & 12 & 0.36 & 22 \\
\hline Hidalgo & -0.20 & 32 & 1.10 & 9 & 0.33 & 25 \\
\hline CORRELACIÓN DE RANGO(SPERMAN) & & & & & \\
\hline C** Y (VA/L)*: 0.71 & & & 0.11 & & 0.65625 & \\
\end{tabular}

Fuente: Elaboración propia con datos del Censo Económico 2009, INEGI.

Como se muestra en la tabla 15, se observa la posición en que se colocó cada estado de la República Mexicana en la comparación de la Competitividad, Productividad y Salarios de la MiPyME, donde 1 significa que es el estado más competitivo y 32 el menos competitivo. Así mismo, se calculó las Correlaciones de rango del Total General de los Estados; en el caso de $\mathrm{C}^{* *}$ y $(\mathrm{VA} / \mathrm{L})^{*}$ : 0.71 ; y $\mathrm{C}^{* *}$ y $(\mathrm{W} / \mathrm{L})^{*}$ : 0.65625 existe una correlación positiva ya que los valores se acercan a 1 , en el caso de $(\mathrm{VA} / \mathrm{L})^{*} \mathrm{Y}(\mathrm{W} / \mathrm{L})^{*}$ : 0.11 ; como el valor se acerca a 0 quiere decir que no hay correlación lineal.

\section{CONCLUSIONES}

Tras el análisis de los resultados obtenidos en el trabajo de investigación, se puede destacar que los objetivos específicos planteados fueron alcanzados satisfactoriamente, lo que conlleva al logro objetivo general.

El estudio del instrumento de recolección de datos, arrojo los siguientes resultados:

- Los directivos de las Asociaciones Cooperativas Banco Comunales, no cuentan con un perfil académico apto para gestionar eficientemente. Sin embargo, muestra gran interés por aprender, y capacitarse, para poner en práctica el Sistema de Información Financiera. Por otro lado, los objetivos del Banco Comunal se ven afectados, debido a la carencia del conocimiento básico de la misión, visión, y normativa legal, es por ello, se le dificultaría guiar a los Banco Comunales en el presente y en el futuro para el logro de los objetivos.

- La información financiera es manejada la mayoría de las veces por integrantes de la directiva del Banco Comunal, y poco son los casos que le envían los papeles de trabajo a un Contador Público Colegiado, mientras que la actualización de la información financiera es realizada al final de la ejecución del presupuesto, lo cual genera limitaciones por no tener la información financiera en el momento oportuno para la toma de decisiones. 
- Las Asociaciones Cooperativas Banco Comunal del Municipio Díaz no poseen normas y procedimientos para la realización de las actividades.

- La falta de comunicación con los entes reguladores, aíslan a los Banco Comunales de información novedosa o de nuevas providencias. El único medio por el cual se comunican con los órganos reguladores, es por la Alcaldía del Municipio Díaz.

- Todo lo que pueda influir en la razonabilidad de la información financiera es de suma importancia, toda gestión se verá reflejada en los documentos tales como estados financieros, libros contables, presupuestos y conciliaciones bancarias, los cuales deben cumplir con los principios de contabilidad de aceptación general y las normas internacionales de contabilidad, para obtener el informe de rendición de cuentas que será presentado ante los organismos competente como FUNDACOMUNAL Y SUNACOOP.

- La mayor cantidad de los Banco Comunales les ha sido aprobado y han ejecutado más de un proyecto, cumpliendo con su gestión social, sin embargo indicaron haber tenido limitaciones a lo largo de su gestión pública. Se evidenció en los instrumentos aplicados en esta investigación, que entre las limitantes se encuentra la falta de conocimientos en materia financiera.

- Es factible, la implementación del Sistema Integral de Información financiera para Optimizar la Gestión Pública de las Asociaciones Cooperativas Bancos Comunales, debido a que la necesidad de asesoramiento, de información, de apoyo técnico, es evidente. Se pudo concluir que, actualmente los Banco Comunales poseen la mayor cantidad de los recursos necesarios para la implementación de tal Sistema, sin duda

- Los Directivo están completamente interesados en disminuir o eliminar las debilidades diagnosticadas a través de esta investigación, para adquirir bases sólidas que garanticen, resultados óptimos al momento de implementar el modelo propuesto.

\section{REFERENCIAS BIBLIOGRÁFICAS}

Andrade, M. (1996). Sistemas y Procedimientos, Nuevas Tendencias en La Contaduría. Editorial Texto, La Vega Caracas.

Andreu, R., Ricart, J. y Valor, J. (1996). Estrategia y Sistemas de Información, Editorial Mc Graw Hill. Madrid.

Arias, Fidias G. (2006). El Proyecto de Investigación. Introducción a la Metodología Científica. 5ta Edición. Editorial Episteme. Venezuela. 